\title{
DIGITIZATION AND EDUCATIONAL PROCESS ... A CONCEPTUAL APPROACH
}

\author{
Sumaiah Eid ALZABOOT ${ }^{1}$
}

\section{Istanbul / Türkiye}

p. $116-135$

Received: $29 / 11 / 2021$

Accepted: 09/12/2021

Published: 01/01/2022

This article has been scanned $k$

iThenticat No plagiarism detected

\section{Abstract:}

The research aimed to reveal the role of the digitization literature, and the theories that explain it, in associating digitization and educational process, by adopting the qualitative approach, and grounded theory method.

The research findings show that the aspects of approach between digitization and the educational process include five elements: teacher and learner, curriculum, educational tools, learning environment, and educational strategies.

The most prominent recommendation is that officials should understand the importance of planning in the use of digitization technology, with the involvement active stakeholders in the field of education. Experts and researchers should be interested in conducting more researches about digitization and educational process.

Key words: Approach, Digitization, Educational Process, Digital Education, Digital Transformation.

http://dx.doi.org/10.47832/2717-8293.15.9

(iD) Prof.Dr. , Rimar Academy, Jordan, alusool2013@gmail.com, https://orcid.org/0000-0002-4012-1259 


\section{الرقمنة والعملية التعليمية .... مقاربة مفاهيمية}

\section{2 سمية عيد زعبوط}

الملتخص:

هدف البحث إلى الكشف عن دور أدبيات الرقمنة، والنظريات المفسرة لها، في المقاربة بين الرقمنة والعملية

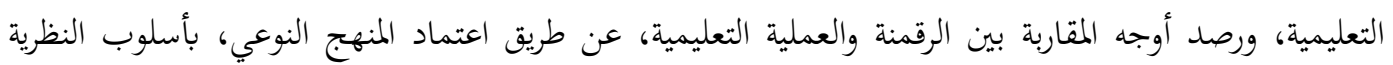
المجذرة. وأظهرت أبرز النتائج أن أوجه المقاربة بين الرقمنة والعملية التعليمية وُّجدَت في خمسة محاور هي: المعلم والمتعلم، المنهاج، والوسائل التعليمية، والبيئة التعليمة، والاستراتيجيات التعليمية.

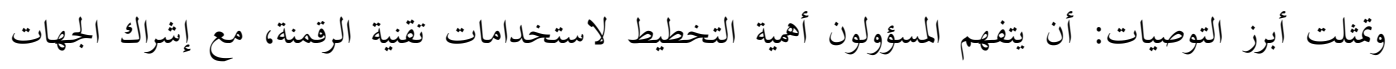

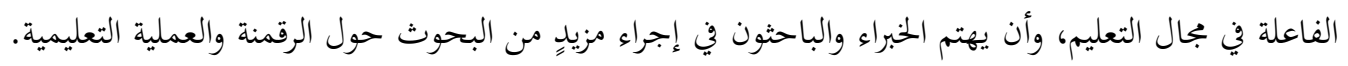

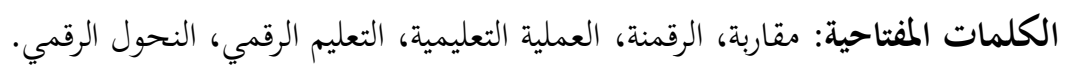

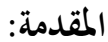

لم أجد أفضل من التوجه إلى يوميات إحدى الأسر كي تكون مدخلاً فيه شيء من الواقعية، فبينما كان الأب ينجزُ بعض كأل

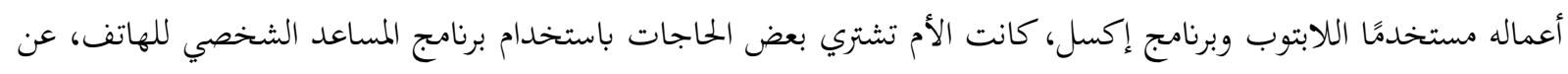
طريق السوق الإلكتروني، ويشارك الابن الأكبر في إحدى الدورات التدريبية عبر إحدى المنصات، أما الابن الأصغر فإنه يتلقى

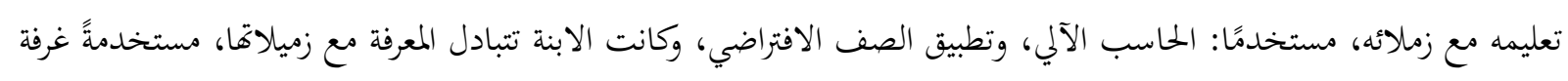

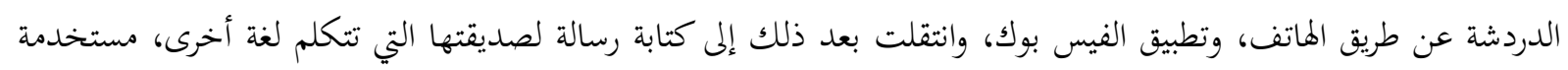
الجهاز اللوحي للترجمة.

يُمكن القول أن هذا الجزء من حياة هذه الأسرة، أصبح مألوفًا لدى كثيرين، إذ أصبح التعلمُ، والعملُ، و واللعبُ، وتبادل

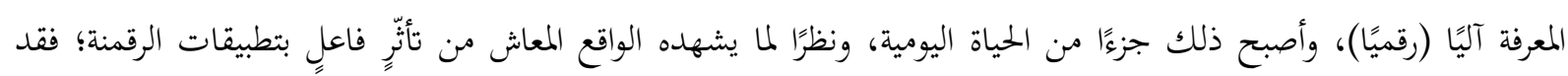

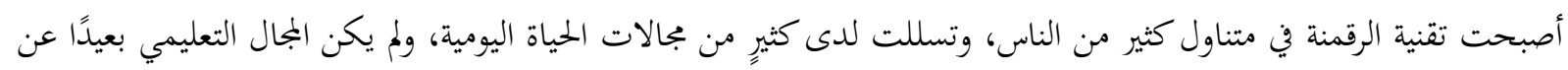
ذلك؛ بل أسهمت الرقمنة في تحقيق مزيدٍ من التحولات الرقمية، وفي تحقيق مزيدٍ من المعرفة العلمية التعلّمية. وقد يُحدثُ هذا المزيد من التحولات الرقمية، والتسارع الذي يُلازمها، خلطًا في المفاهيم المنبثقة عن الرقمنة؛ ما يُوثنر سلبًا في العملية التعليمية في ظل الرقمنة، الأمر الذي يُؤكد الحاجة إلى المقاربة المفاهيمية بين الرقمنة والعملية التعليمية.

مشكلة البحث:

تكمن مشكلة البحث فيما يُلاحظ عن طريق الاطلاع، والبحث في بعض الأدبيات، والنظريات التي تم رصدها، إذ لوحظ

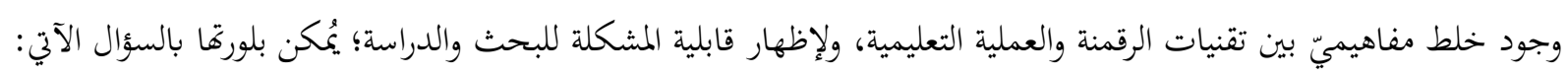

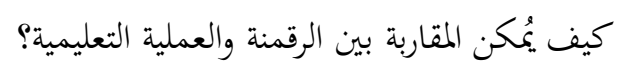


أسئلة البحث: يُمكن توضيح مشكلة البحث بالإجابة عن الأسئلة الآتية: السؤال الرئيس: ما إمكانية المقاربة بين الرقمنة والعملية التعليمية؟ وتتبلور إمكانية المقاربة بالإجابة عن الأسئلة الفرعية الآتية: 1. ما دور أدبيات الرقمنة في المقاربة بين الرقمنة والعملية التعليمية؟

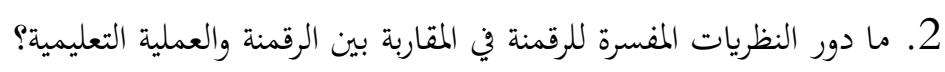

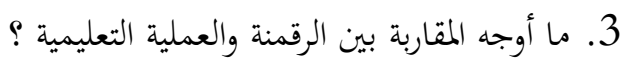

$$
\begin{aligned}
& \text { أهداف البحث: يُمكن الإجابة عن أسئلة البحث بتحقيق الأهداف الآتية: } \\
& \text { 1. الكشف عن دور أدبيات الرقمنة في المقاربة بين الرقمنة والعملية التعليمية . } \\
& \text { 2. الكشف عن دور النظريات المفسرة للرقمنة في المقاربة بين الرقمنة والعملية التعليمية. } \\
& \text { 3. رصد أوجه المقاربة بين الرقمنة والعملية التعليمية. }
\end{aligned}
$$

\section{أهمية البحث: تمثلت أهمية البحث بالآتي:}

تلبية البحث لأحد محاور المؤتمر العلمي الدولي للدراسات المعاصرة في العلوم الاجتماعية، ألا وهو: محور التربية والتعليم.

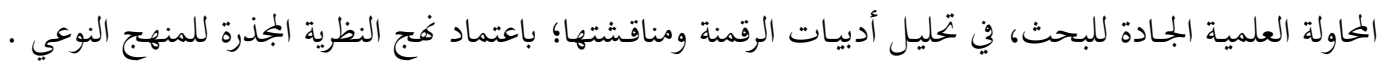
انتهاج البحث فه المقاربة بين الرقمنة، والعملية التعليمية؛ عن طريق استقراء بعض الأدبيات والبحوث التي تتعلق بالرقمنة والحياة الرقمية؛ الأمر الذي يُضفي تنوعاً مهماً في الطرح.

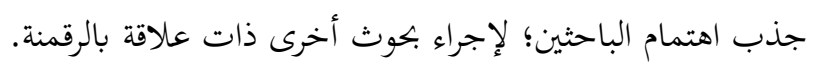

منهجية البحث: تم اعتماد النظرية المجذرة بوصفها إحدى أساليب البحث النوعي، للمقاربة بين الرقمنة، والعملية التعليمية،

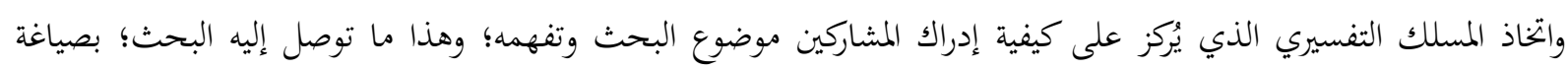

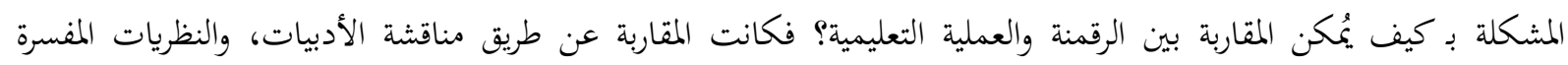

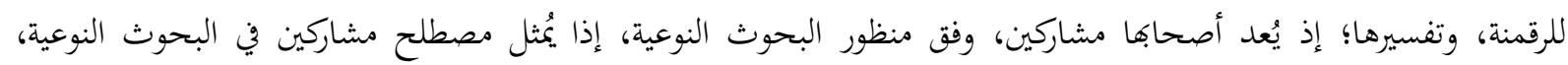

$$
\text { مصطلح العينة في البحوث الكمية. }
$$

ولتوضيح عملية المقاربة، اعتُمَدَ النموذج المطور من قبل ماكسويل (Maxwell) عام (1998م)، بمكوناته الخمسة: أسئلة البحث، أهداف البحث، الإطار المفاهيمي، المنهج، النتائج، على النحو الآتي: 


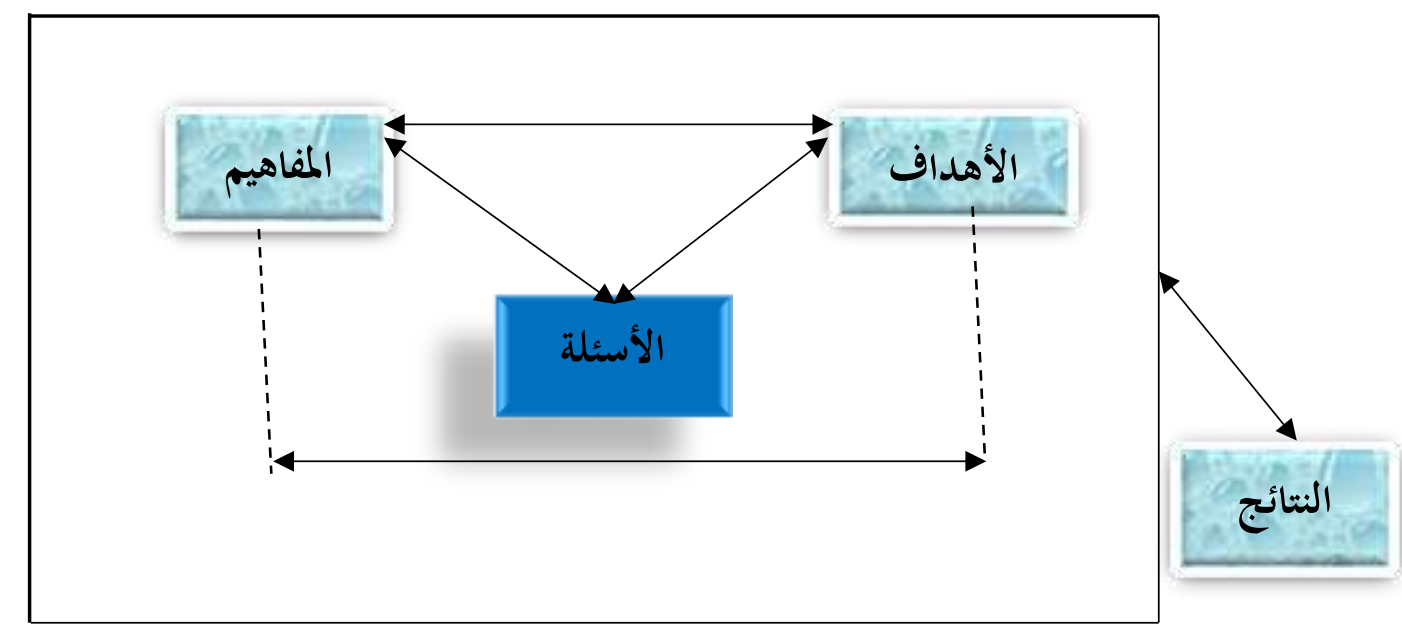

الشكل (1): نموذج ماكسويل

يُلاحظ من الشكل (1) أن أسئلة البحث هي نقطة الانطلاق بالنسبة للبحث النوعي؛ ويتبين من السهم الموصول(ب)

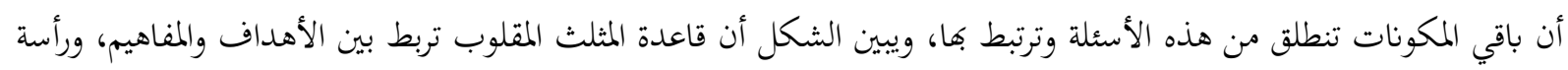
مرتبط بأسئلة البحث؛ ما يُوكد أن أسئلة البحث تستدعي صياغة الأهداف، وتُشَّل الإطار المفاهيمي؛ لتحقيق الأهداف. أما المثلث في أسفل الشكل فهو يربط بين المنهج، والنتائج، ورأسه في الأعلى مرتبط بالأسئلة ما يُوكد أن أسئلة البحث

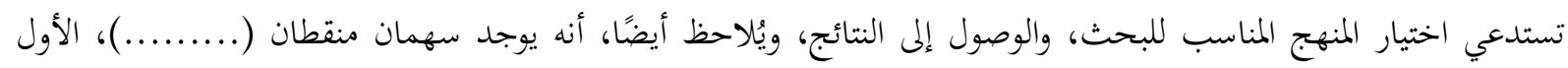

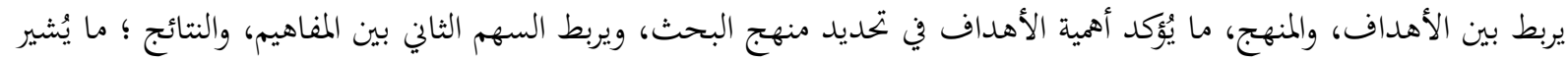
إلى أن مناقشة النتائج وتفسيرها، ينبغي أن تكون في ضوء المفاهيم المعتمدة في البحث؛ لتحقيق الأهداف.

المواد المشاركة في البحث:

يُطلق على عينة البحث النوعي مسمى المواد المشاركة أو المشاركين؛ سواء أكانوا أفرادًا أم غير ذلك، نظرًا للتفاعل المشترك

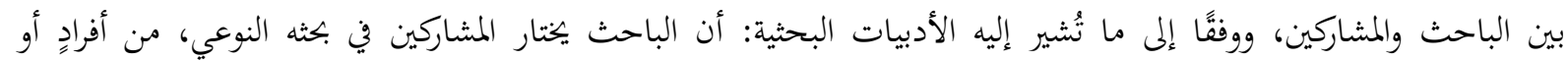
أدبيات، أو وثائق بطريقة مقصودة، بناءً على تقديره وتصوره أن ذلك يُسهم في تحقيق أهداف البحث، ويُجيب عن أسئية أسئله. (الفقيه، 2017) . لذا اختيرت خمس أدبيات ونظريتان بطريقة العينة القصدية؛ لتمثل المواد المشاركة للبحث الحالي.

\section{المصطلحات العلمية للبحث:}

مقاربة: هي أساس نظري يتكون من مجموعة من المبادي؛ وهي الطريقة التي يعتمدها الدارس، أو الباحث؛ من أجل

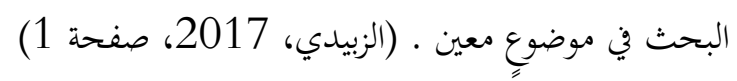

المقاربة إجرائيًا هي: الأسس والمفاهيم التي تحتوى على سمات مشتركة، ويتم التوصل إليها بعد مناقشة المعلومات وتحليلها. الرقمنة: هي عملية تحويل الإجراءات والأنشطة من أنشطة تقليدية، إلى أنشطة رقمية، باستخدام الأجهزة الآلية، والبرمجيات، والتطبيقات الرقمية ـ (الحوسي، 2021، صفية صفحة 1) 
الرقمنة إجرائيًا هي: المهمة التي تتعلق بالحاسب الآلي، والتطبيقات المنبثقة عنه، وتُشير هذه المهمة إلى تحويل المعلومات،

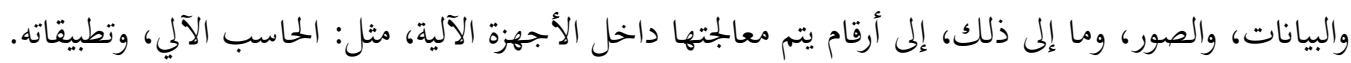

العملية التعليمية: هي بجموعة منظمة من الأنشطة والإجراءات، تحدف إلى تلبية الحاجات التعليمية وتتضمن العملية

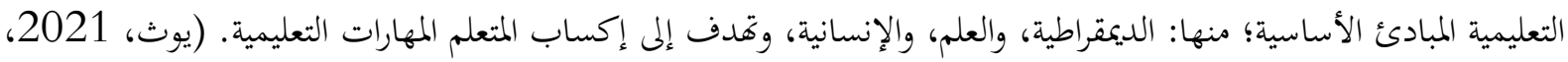

صفحة 1)

التعليمية إجرائيًا هي: أنشطة علمية مدروسة، تمدف إلى إكساب المتعلم المعرفة في مراحلٍ مختلفة؛ باعتماد الأساليب المناسبة لكل مرحلة، ومن أهم عناصرها المعلم، والمتعلم، والمنهاج.

التعليم الرقمي: هو تقديم محتوى تعليمي إلكتروني عبر الوسائط المعتمدة الموجودة على الحاسب الآلي، وشبكاته، إلى

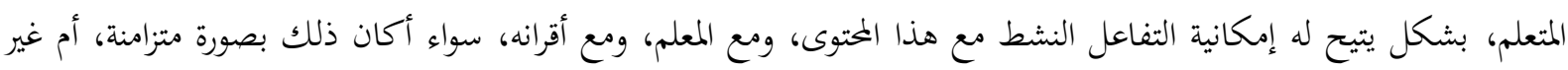

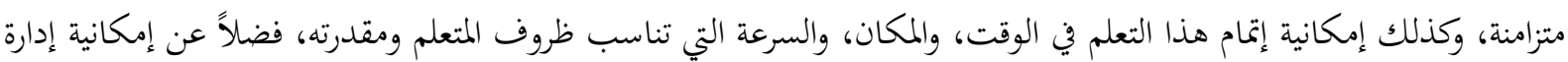

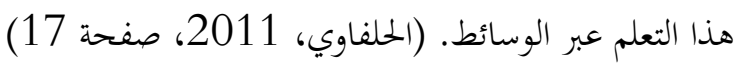

التعليم الرقمي إجرائيًا هو: التعليم الذي يعتمد استخدام جهاز الحاسب الآلي، وبرجياته، أو اللابتوب، أو الشبكة الإلكترونية، وتطبيقاةا، ويشمل التعليم الافتراضي، والتعليم الشبكي، والتعليم الإلكتروني، والجانب الإلكتروني من التعليم عن بعد. التحول الرقمي هو: الاستثمار في الفكر وتغيير السلوك؛ لإحداث تحول جذري في طريقة العمل، عن طريق الاستفادة من

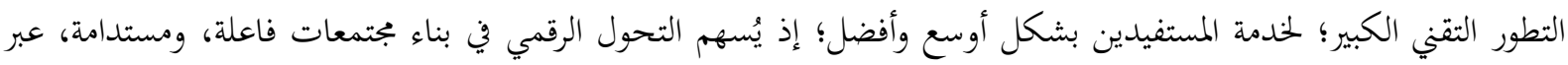
تحقيق تغيير جذري في خدمات مختلفة الأطراف من مستهلكين، وموظفين، ومستفيدين، مع تحسين بتحاركم وتطويرها، عبر سلسلة من العمليات، وإعادة صياغة الإجراءات اللازمة للتفعيل والتنفيذ. (المفضي، 2021، معني، صفينة التحول الرقمي إجرائيًا هو: عملية استبدال الأساليب والوسائل، والخدمات المستخدمة، والسلوكات الممارسة في التعليم، والعمل، في الواقع الحالي، إلى أساليب العالم الرقمي، ووسائله وخدماته، ومن أمثلة ذلك: الحوسبة السحابية، بوصفها تحول رقمي،

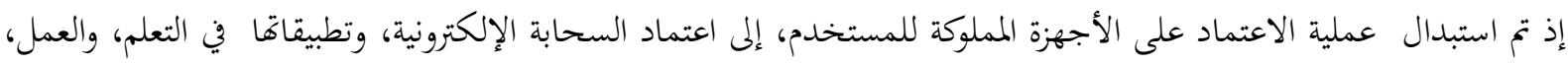
والتسوق، وغير ذلك.

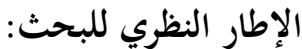

تضمن الإطار النظري للبحث أربعة محاور هي: الرقمنة، وأدبيات الرقمنة، والنظريات المفسرة للرقمنة، ومناقشة المحاور وتفسيرها، على النحو الآتي:

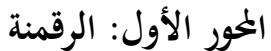

تُشير الرقمنة لغويًا في المعجم الوسيط، إلى أها من الرَّم، بمعنى الخط العريض، ويُقال جاء بالرقم أي الكثير وفي علم

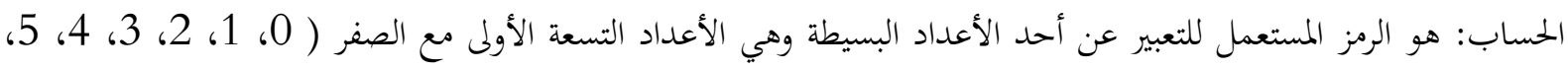

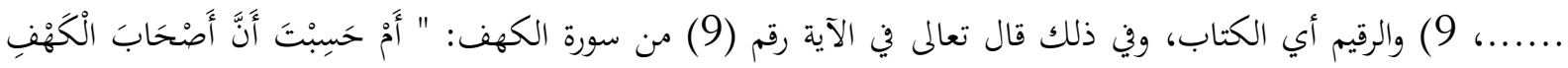

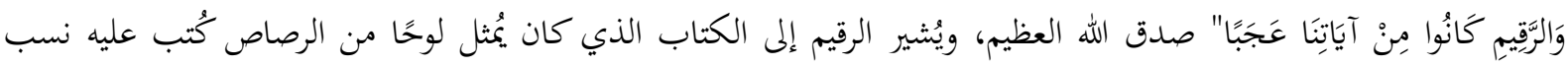

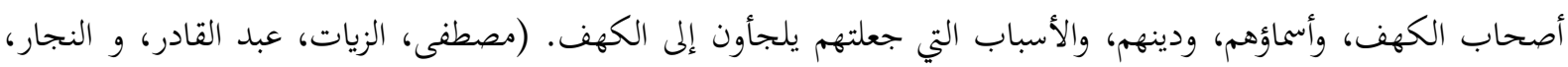
بدون تاريخ، صفحة 367) 
بناءً على مفهوم الرقمة ؛ فإن تقنياتا دخلت في مجالات الحياة كافة، منها: الاجتماعية، والاقتصادية، والزراعية، والتربوية،

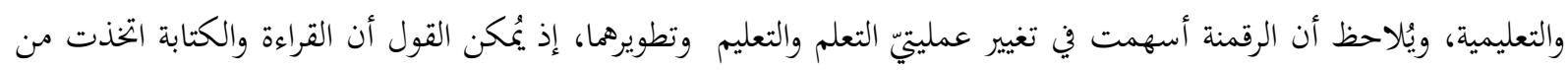

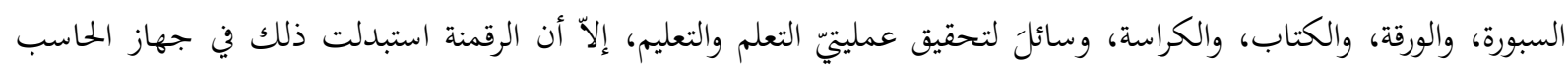
الآلي، وبربجياته، وفي تطبيقات السحابة الإلكترونية، والشبكة الإلكترونية. (الزعبوط، وابك، 2021) ومن منطلق أن الحاسب الآلي اعتمد في البداية على الأرقام فيما يُسمى بالخوارزميات، التي يعود أصلها إلى عالم الرياضيات

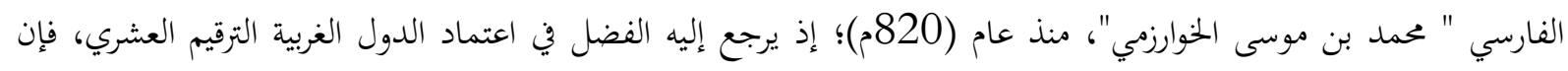

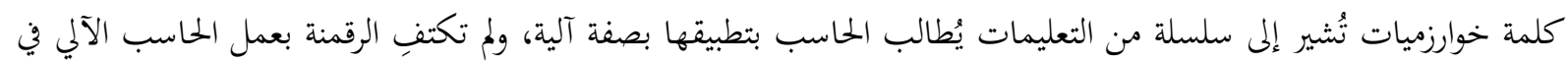

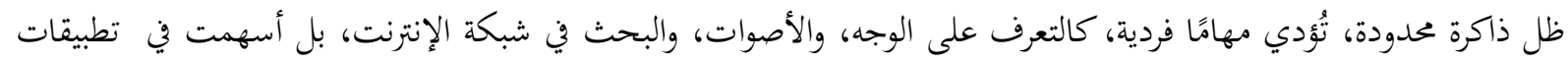

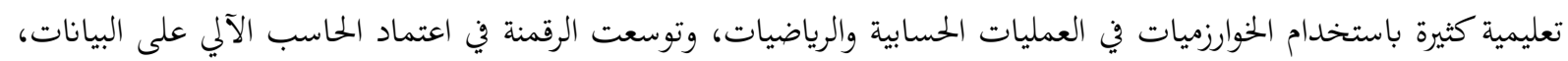

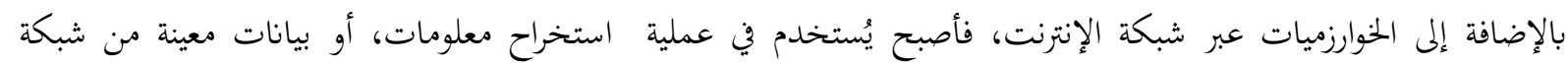

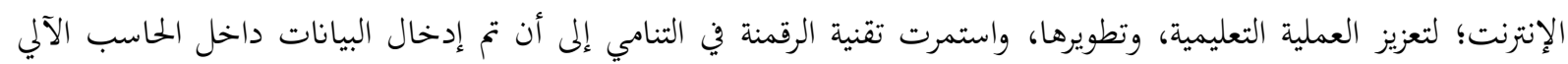

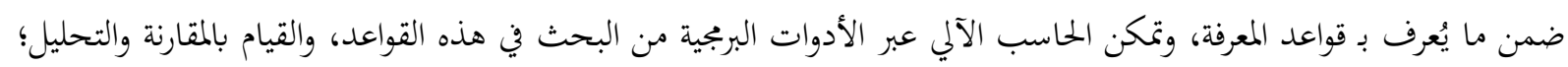

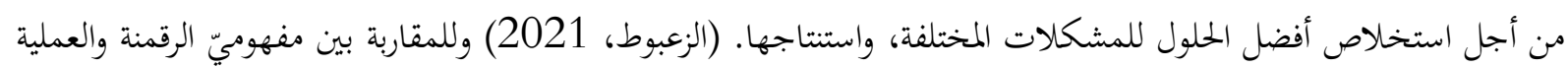
التعليمية، ينبغي البحث في أدبيات الرقمنة والنظريات المفسرة لها، عبر محاور البحث.

الخور الثاني: أدبيات الرقمنة

ركزت خطة عمل المفوضية الأوروبية على العملية التعليمية الرقمية عبر إعادة التعليم والتدريب للعصر الرقمي، وأظهرت

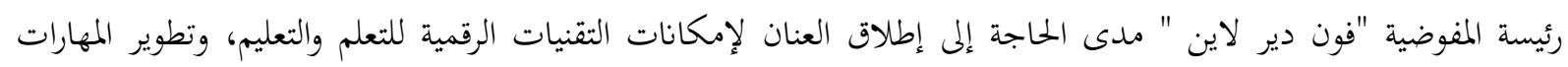

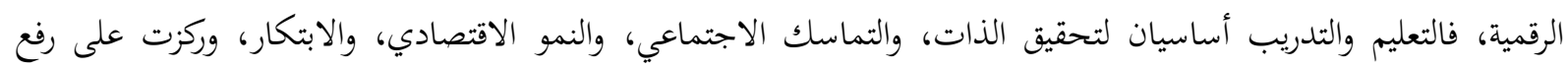

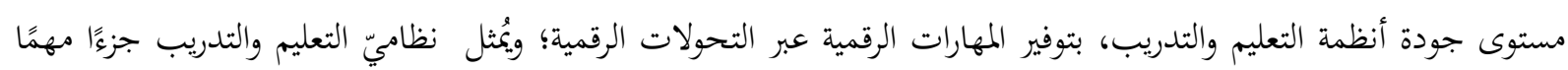

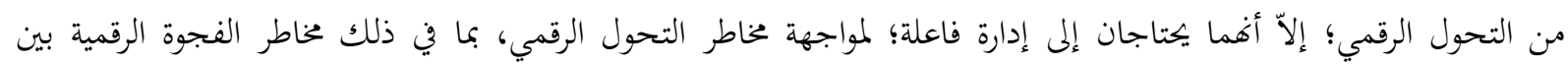

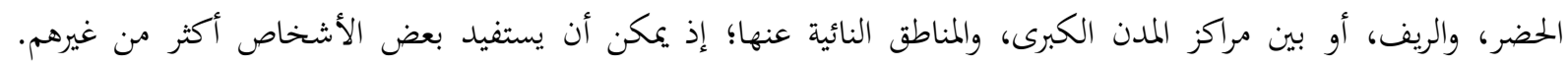

(Commission, 2021)

وركزت الخطة أيضًا على جانبين مترابطينْ للعملية التعليمية الرقمية هما: (Commission, 2021)

الأول: نشر مجموعة واسعة من التقنيات الرقمية (التطبيقات والمنصات والبرجيات)؛ لتحسين نطاق التعليم والتدريب وتوسيعه، إذ إن التعليم عبر الإنترنت، والتعليم عن بعد، والتعليم المدمج أمثلة محددة لكيفية استخدام

$$
\text { تقنية الرقمنة لدعم عملتيّ التعليم والتعلم. }
$$

الثاني: تزويد المتعلمين كافة، بالكفاءات الرقمية (المعرفة والمهارات والمواقف)؛ للعيش، والعمل، والتعلم، والازدهار في عالم تتوسطه التقنيات الرقمية بشكل متزايد.

وركزت خطة العمل الأوروبية على التغيير الرقمي طويل المدى في التعليم والتدريب، الذي يشمل تحسين مهارات القراءة

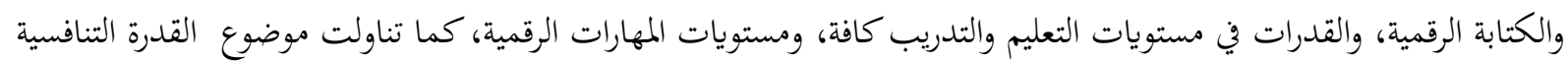

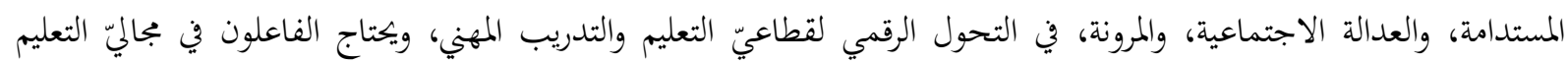


والتدريب إلى التفكير بشكل استراتيجي في كيفية دمج التقنيات الرقمية في التعليم والتدريب، ويُشير التفكير بشكل استراتيجي إلى كيفية دمج التقنيات الرقمية في التعليم والتدريب إلى الآتي: (Commission, 2021)

التحول الرقمي: إن تحويل التعليم للعصر الرقمي مهمة تشمل أفراد المجتمع كافة: بحيث يشترك التربويون، والقطاع العام،

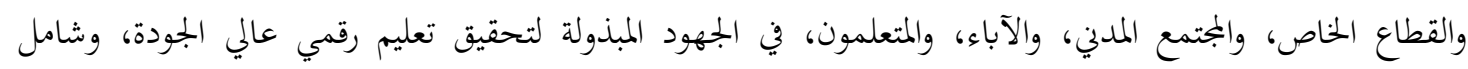

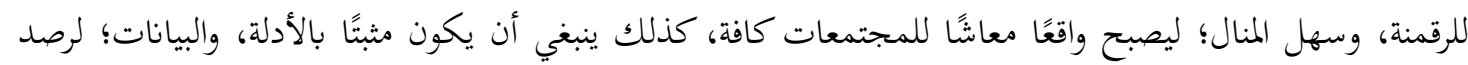

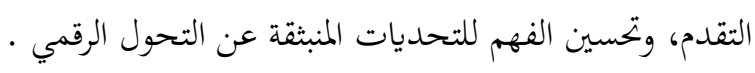

الاستثمار المناسب: ينبغي أن يكون الاستثمار في الاتصالات، والتجهيزات، والقدرات، والمهارات التهات التنظيمية، لتتمكن

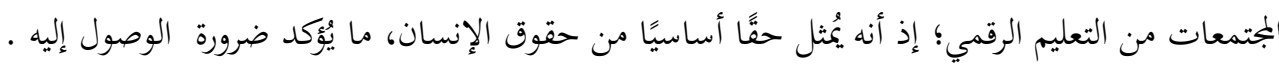

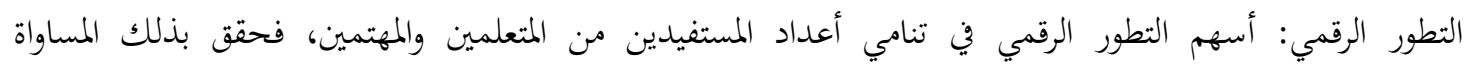
والشمولية، وتناميًا في المهارات الرقمية، ونشر أنظمة شاملة يمكن الوصول إليها رقمياً؛ إذ إن الافتقار إلى المهارات

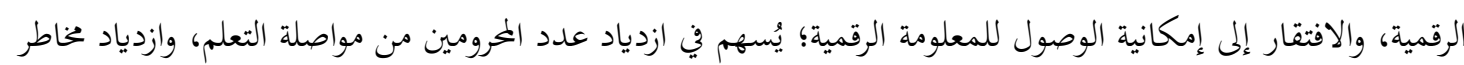
الفقر والحرمان، كذلك يُسهم في تنامي الأمية الرقمية.

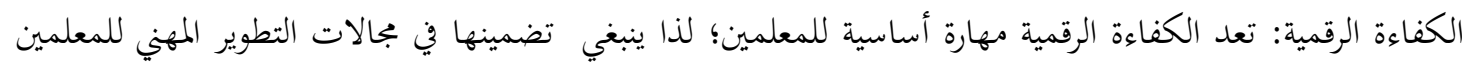

كافة. محو الأمية الرقمية: يُعد محو الأمية الرقمية من ضروريات الرقمنة؛ إذ تدخل الحاسبات والخوارزميات في الأنشطة اليومية

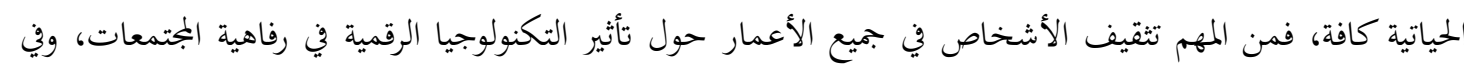

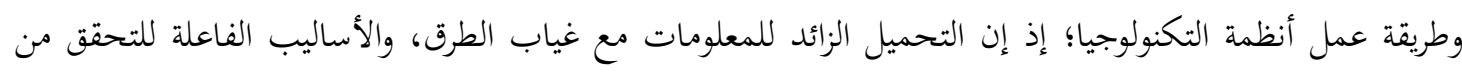

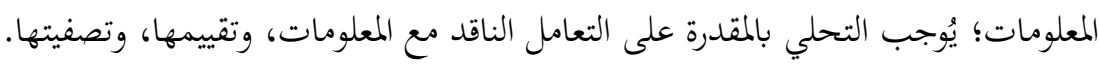
وأظهرت إحدى الأدبيات تصورات طلبة المرحلة الجامعية حول التعليم الإلكتروني، إذ اتخذ الباحثون عينة تكونت من

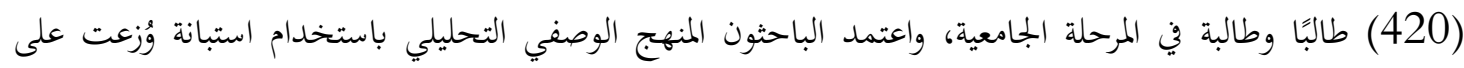

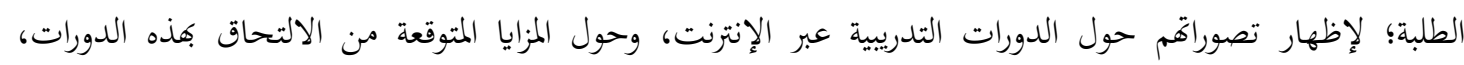

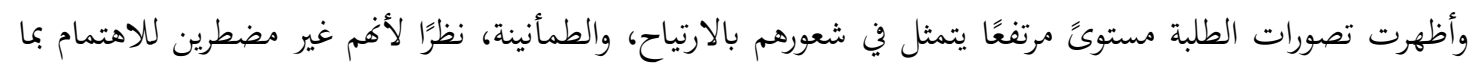

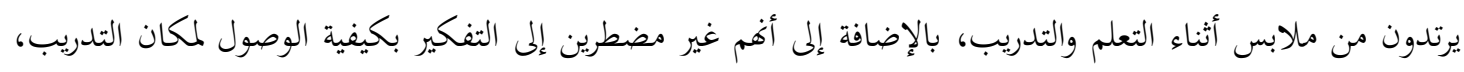

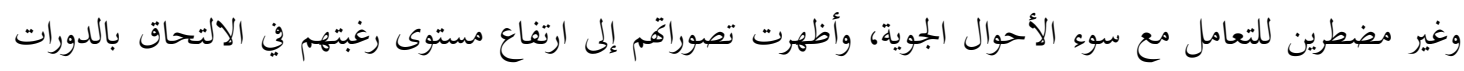

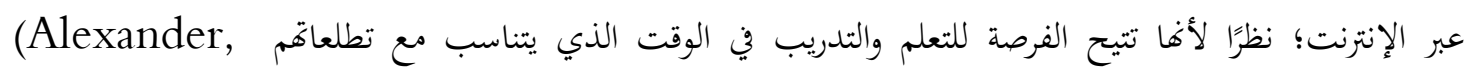
.Truell, \& Zhao, 2012)

كما أظهرت تصورات الطلبة بعض العيوب الملازمة للدورات التدريبية الرقمية، التي تتعلق باحتمال المماطلة، وقلة فهم

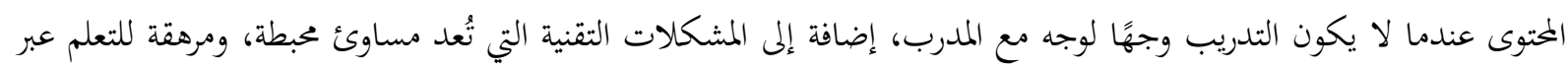

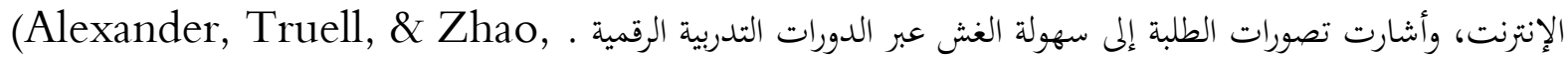

وأوردت إحدى دراسات معهد تونغ فانغ للتصميم في تايوان أن التعلم الرقمي جاء في البداية ليحمل مسمى التعليم

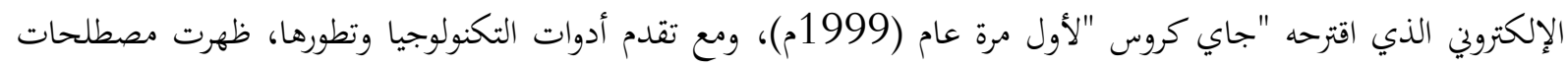

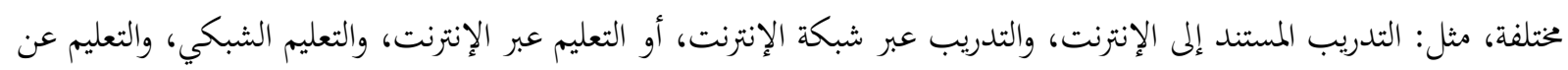


بعد، ويُعد التعليم الرقمي شاملاً لمذه الأنواع، وفي العصر الذي تتدفق فيه المعرفة والمعلومات بسرعة؛ يغطي تطبيق التعليم الرقمي مجالات الحياة كافة. (Ming, Huang, \& Kuang, 2017) بناءً على ذلك، فقد اقترحت الجمعية الأمريكية للتدريب والتعليم (ASTD) أن التعليم الرقمي يُشير إلى أنه عملية

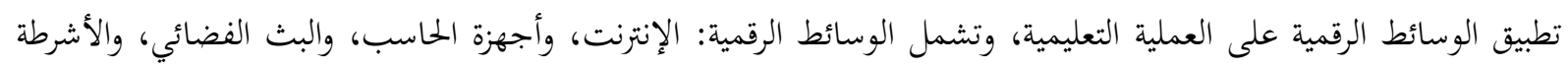

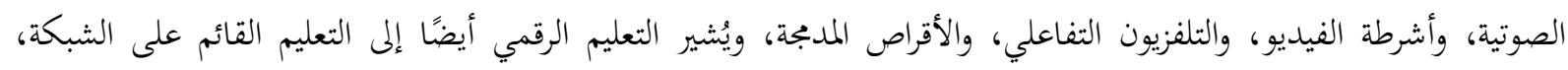

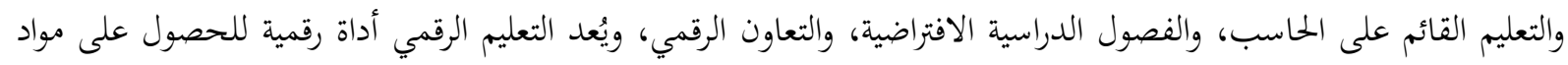

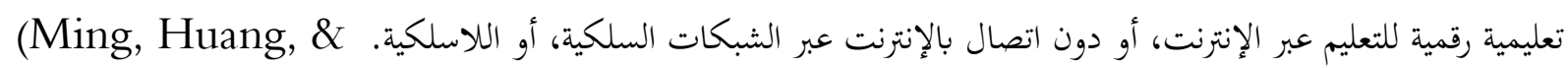

Kuang, 2017)

وأظهرت الدراسة: أن التعلم الرقمي يُسهم في تعزيز ممارسات إيمابية تُحفز على التعلم بشكلٍ أفضل، مقارنة بما يقدمه

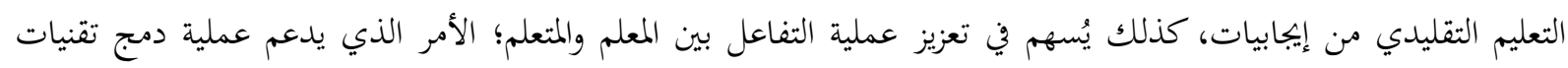

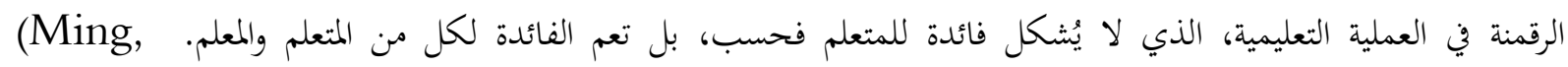

Huang, \& Kuang, 2017)

وأظهرت إحدى أدبيات اليونسيف عام (2021) أن المجتمعات منخفضة الدخل، وذات الدخل المتوسط تواجه عددًا لا

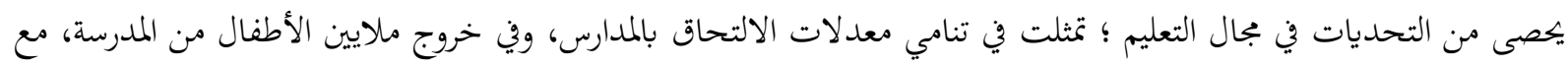

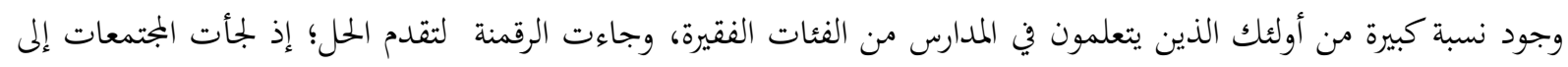

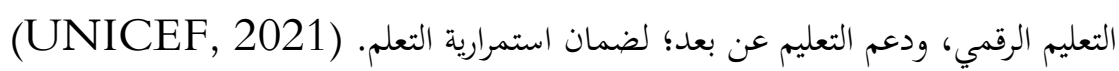

وحول تطبيق حلول التعليم الرقمي في البلدان المنخفضة الدخل، والبلدان مرتفعة الدخل، أظهر هذا التطبيق وعدًا كبيرًا في تحسين نتائج التعلم، عندما يتم دججه في عمليات التعليم؛ إذ يؤدي دمجه في التعليم إلى تسهيل المرونة في التعليم، وإلى تزويد المتعلمين عملية التحكم في تعلمهم، وتشجيع تنمية الكفاءات المعرفية. (UNICEF, 2021) وقد ثبت أن حلول التعلم الرقمي تجذب المتعلمين عاطفياً، إذ يتضح أن مواقف المتعلمين، ومتعتهم واهتماماتم تزداد بتحاه

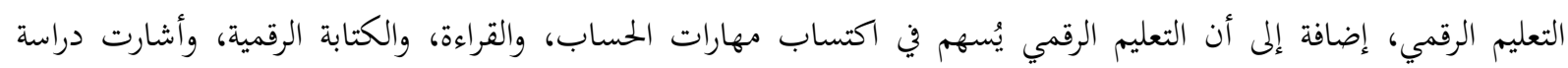

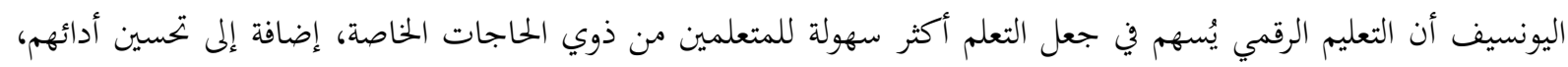
وتحسين مهارات القراءة لدى الذين يعانون من عسر القراءة، وتعزيز مهارات الرياضيات للصم، وضعئ وضعاف السمع، والمكلكوفين.

(UNICEF, 2021)

واهتمت إحدى الأدبيات بموضوع منصات التعليم الرقمي، وذلك عبر دراسة نقدية لهذه المنصات، إذ أوردت أن التعليم

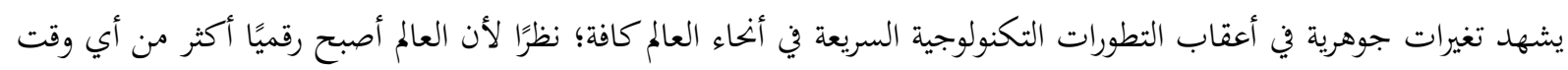

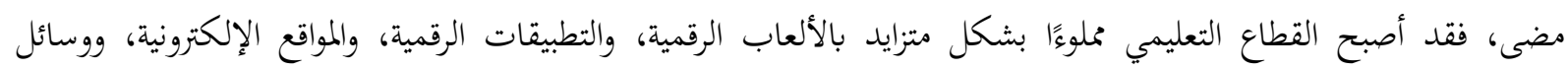

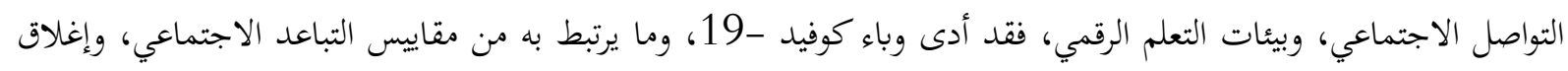

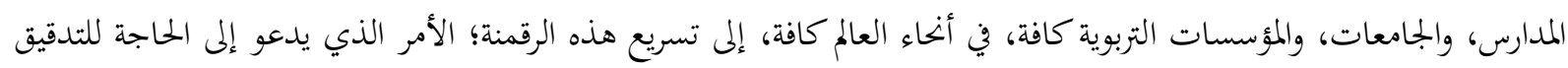
الدقيق حول كيفية إعادة تشكيل هذه الرقمنة ودبجها في البيئات التعليمية. (Decuypere, Landri, \& Grimaldi,

كذلك، انتشرت المنصات، وتنوعت أشكالها، وأصبحت شركات التكنولوجيا العالمية، والمحلية على حد سواء، من البيئات

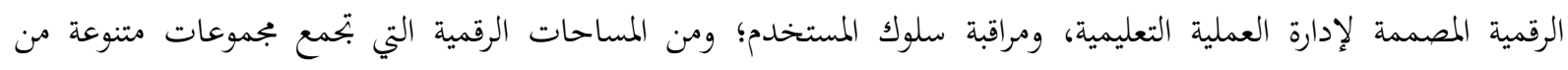


الوظائف إلى واجهات أكثر تفردًا، وأشارت الدراسة إلى منصات رقمية متخصصة لكل مجال من بجالات الحياة، مثل: المجال

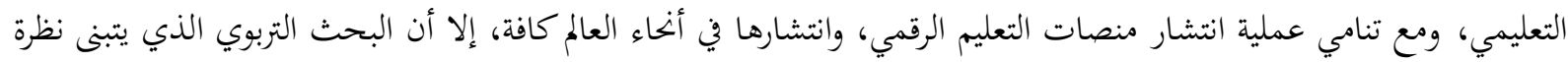
نقدية لمثل هذه المنصات لا يزال محدودًا. (Decuypere, Landri, \& Grimaldi, 2021) وأشارت الدراسة إلى توافر النظام الأساسي لهذه المنصات عبر الإنترنت، فهو يمُثل بنية رقمية قابلة للبرمجة، ومصممة لتنظيم التفاعلات بين المستخدمين، والشركات، والهيئات، وما إلى ذلك، كذلك هو موجه بأسلوب منهجي؛ ليقوم بالمعالجة الحسابية، وتداول البيانات، وتبادل المعلومات، وتحقيق الدخل من بيانات المستخدم؛ لذا فالحاجة ماسة إلى المنصات الرقمية، لأهما أصبحت راسخة في الحياة الاجتماعية بشكل عام، وفي المجال التعليمي بشكل خاص، ما أدى إلى تبنّ المؤسسات التعليمية بجموعة متنوعة من المنصات العالمية التي تتعلق بالتعليم. (Decuypere, Landri, \& Grimaldi, 2021) وتُعد المنصات الرقمية معماريات رقمية؛ فهي تعمل كأبراج زمانية، ومكانية، وتُمكّن المستخدم من عرض أنشطته، وتتكون المنصات الرقمية من نوعين مختلفين من الواجهات: واجهات المستخدم الرسومية، وواجهات بربجة التطبيقات، وتشتهر المنصات

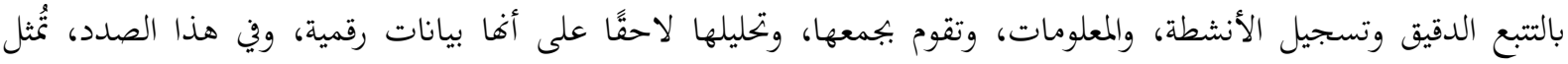

المنصات الرقمية نوعًا جديدًا من البيئات التعليمية. (Decuypere, Landri, \& Grimaldi, 2021) وتعتمد المنصات الرقمية وسائل جديدة لإنتاج قيمة، ما يعني أها تقيس أنشطة المستخدم، ومعلوماته بوصفها بيانات، وتقدم تلك الأنشطة عبر المنصات الرقمية على أفها سلوك يمكن ملاحظته وقياسه، وبذلك فهي تعمل بوصفها قناةً؛ لتسهيل تبادل المحتوى والأنشطة التعليمية، مثل: مجموعة (منصات أمازون)، ومنصة (كلاس رووم)، وكثير من أنظمة إدارة التعلم الشائعة

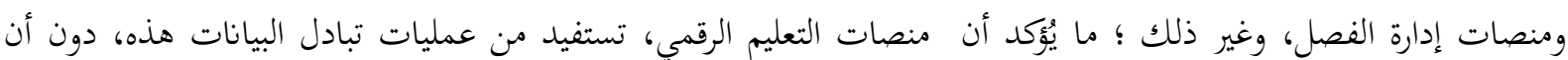

تشارك بالضرورة في إنتاج المحتوى التعليمي بنفسها. (Decuypere, Landri, \& Grimaldi, 2021) ومن المنصات ما يتسم بالكفاءة في تقديم المحتوى التعليمي، ما يعني أها تعد مزودًا للمحتوى المنتج ذاتيًا، مثل: منصة(

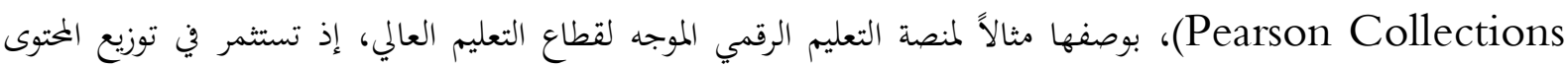

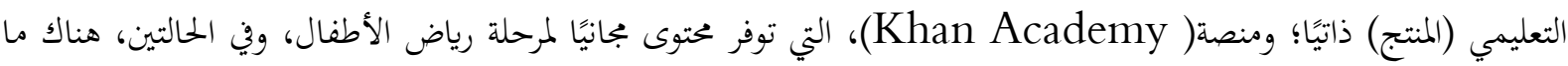

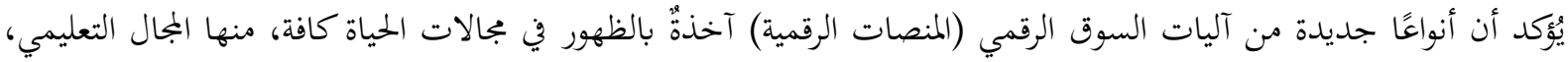

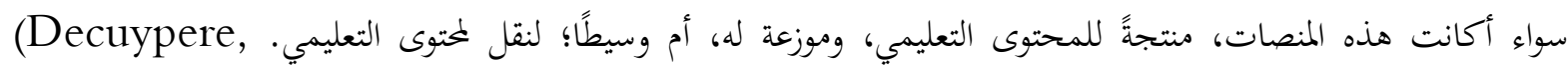
Landri, \& Grimaldi, 2021)

المحور الثالث: النظريات المفسرة للرقمنة إن تبني نظريات تعلم حديثة، يُسهم في تصميم الأنظمة المعرفية والتقنية وتنظيمها؛ ويُمْكّن من صناعة المستقبل القائم على

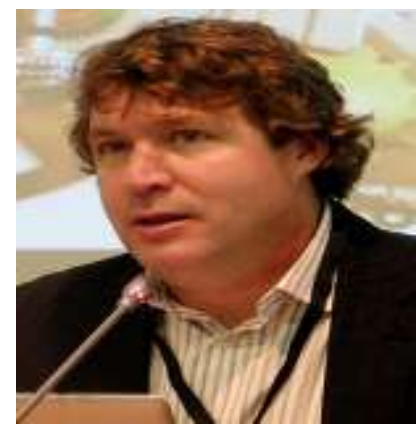

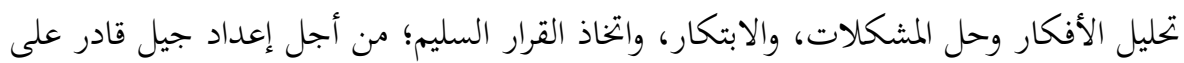

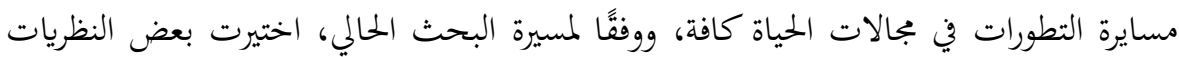
المفسرة للرقمنة. نظرية الاتصال الشبكي لـ جورج سيمنز

وُلََِِ جورج سيمنز عام (1970م) وهو كندي الأصل، وحصل على درجة الدكتوراه في علم النفس من جامعة أبردين عام (2011م)، وطورت أطروحته كنموذج للمعلومات في بمال 
السلوك الفردي في الشبكات الاجتماعية، وانضم سيمنز إلى هيئة التدريس في جامعة تكساس في( أرلينغتون )، بوصفه أستاذًا في

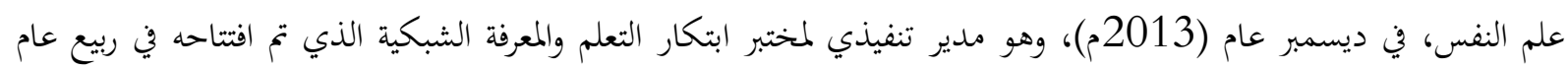
(2014م)، وهو مؤلف لكتاب " معرفة المعرفة" الذي ترجم إلى عدة دمات لغات منها: الإسبانية، والفارسية، والهنغارية.

(Siemens, wikipedia., 2020)

تسعى النظرية الاتصالية إلى توضيح كيفية حدوث التعلم في البيئات الإلكترونية المركبة، وكيفية تأثر التعلم عبر

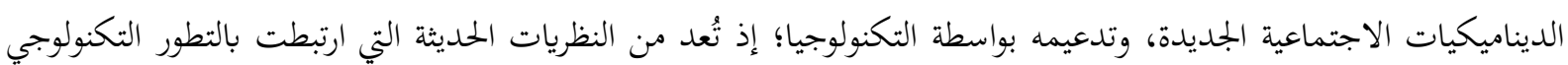

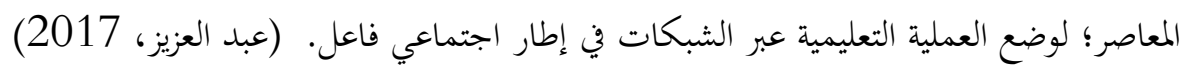
وترتكز النظرية الاتصالية على مقولة أن العصر الحالي، هو عصر قائم على المعرفة في مجالات الحياة كافة؛ ولهذا فالإنسان

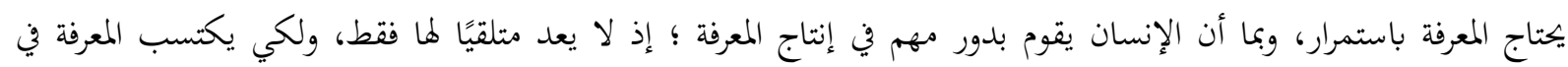

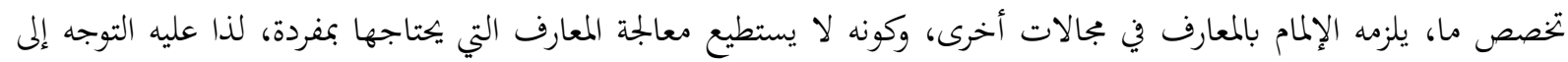

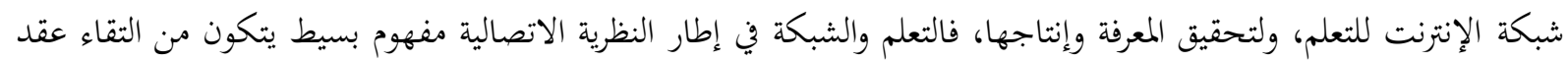

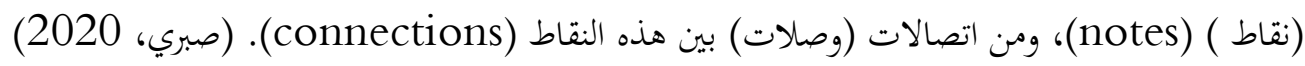
وتُشير نقاط الالتقاء إلى مكونات بشرية، ومكونات غير بشرية، وتتضمن المكونات البشرية المتعلمين، أو الخبراء في مجالات

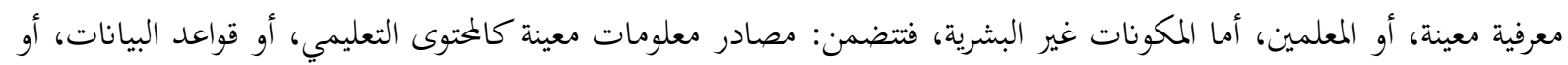

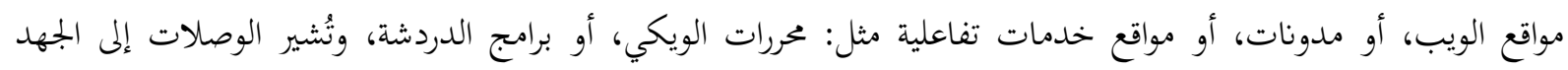
المبذول في عملية التعليم، إذ يربط هذا الجهد التعليمي (الوصلات) بين النقاط المتمثلة بالمكونات البشرية، والمكونات غير البشرية. (صبري، 2020)

ومن مميزات النظرية الاتصالية أها تُسهم في تشجيع الاتصال بين المتعلم والمؤسسة التعليمية، كما أن لها دور في تنمية التبادل والتعاون بين المتعلمين عبر التعلم النشط، وتركز دائماً على إعطاء تغذية راجعة؛ للتأكيد على أهمية الوقت في إنجاز المهمة، لتهات

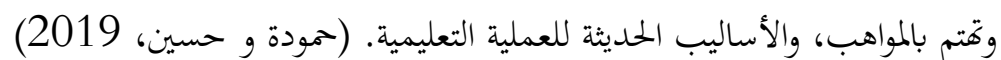

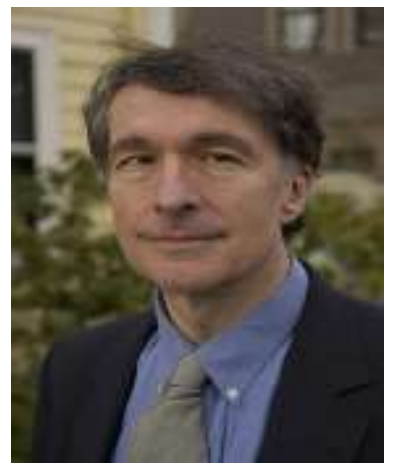

نظرية العقول الخمسة لموارد جاردنر

ولد هوارد جاردنر في 11 يوليو عام (1943م) في سكرانتون بولاية بنسلفانيا، وحصل على درجة الدكتوراة في جامعة هارفارد عام (1971م)، وهو عالم في علم النفس التنموي، واشتهر بنظرية الذكاءات المتعددة؛ إذ كان يعتقد أن المفهوم التقليدي للذكاء يتسم

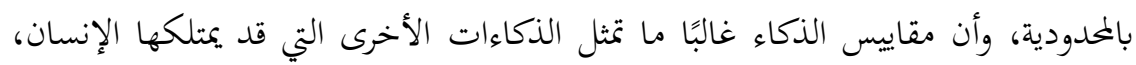

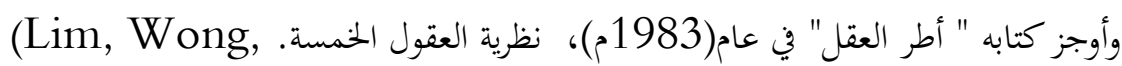
Boon, \& Tan, 2009)

وتُعد نظرية العقول الخمسة من النظريات التربوية الحديثة لهوارد جاردنر الذي أشار عبرها إلى مجموعة القدرات العقلية التي ينبغي أن يتصف بها إنسان القرن الحادي والعشرين، وهي العقل المتخصص، والعقل التركيبي، والعقل المبدع، والعقل المحترم، والعقل

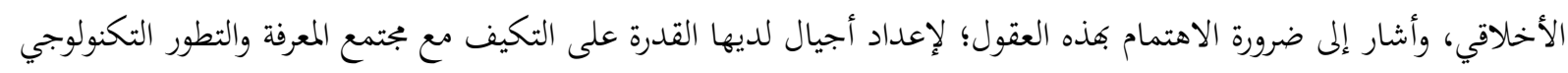
في العصر الرقمي. (Kimberly, 2014) 


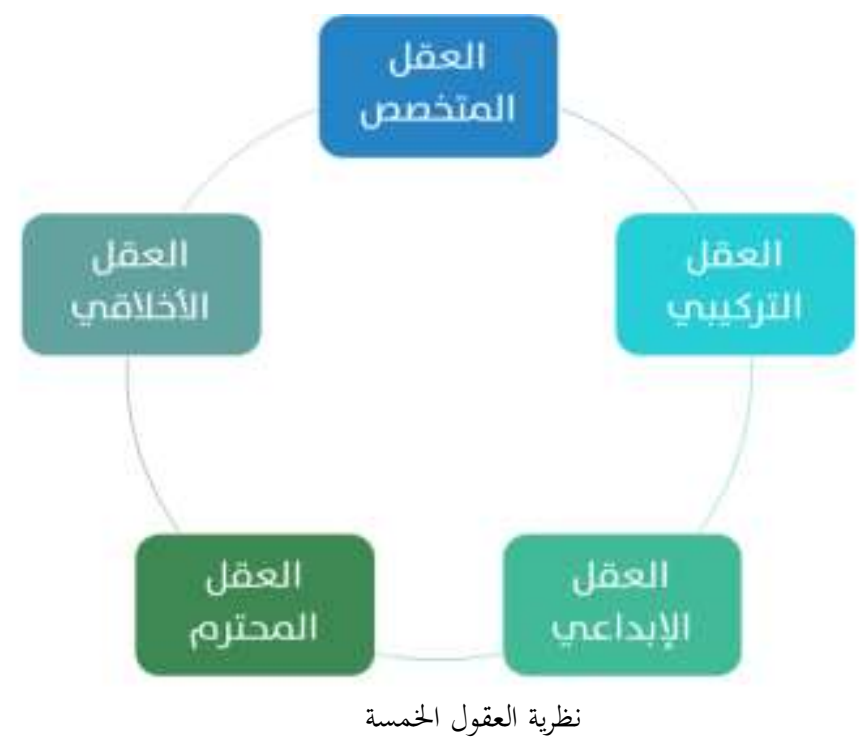

https://shaghf.net/ar/resources/read/khmsh-aqwl-mn-ajl-almstqbl:المصدر

وفي ذات السياق، يُشير العقل المتخصص، أو التخصصي إلى مقدرة المتعلم على تحديد المعارف المهمة، والمعارف غير

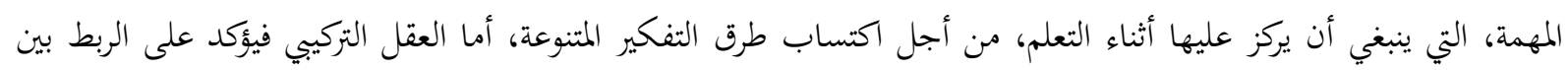

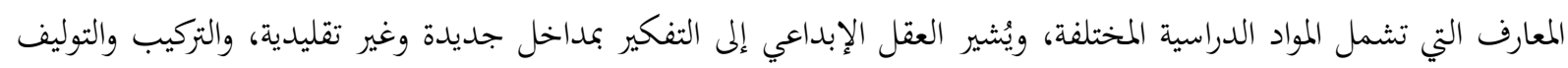

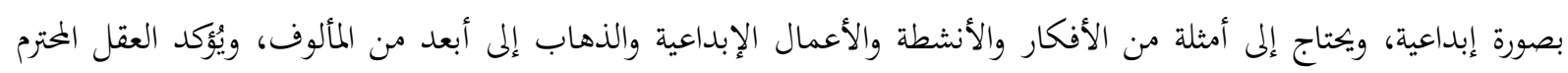

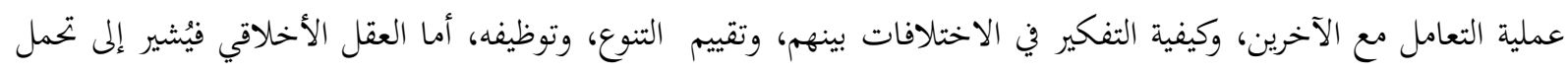

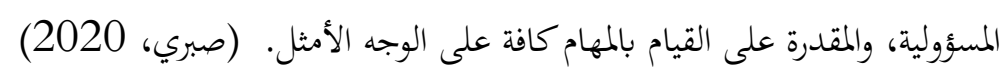

بناءً على ذلك، ينبغي أن تسعى المؤسسات التعليمية إلى تنمية العقول الخمسة لجاردنر للمراحل التعليمية كافة؛ نظرًا لما

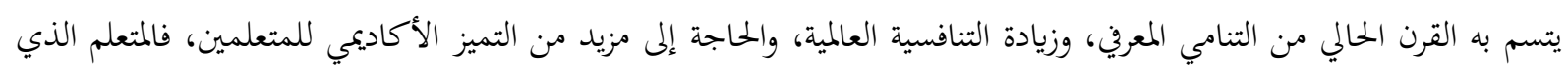

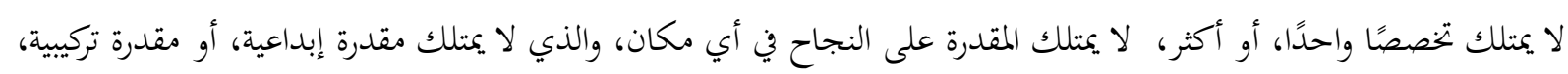

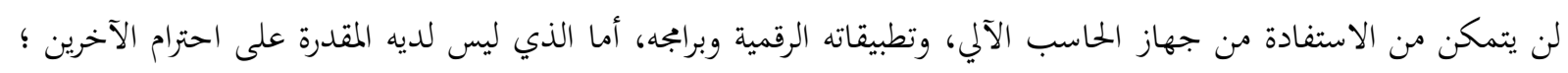

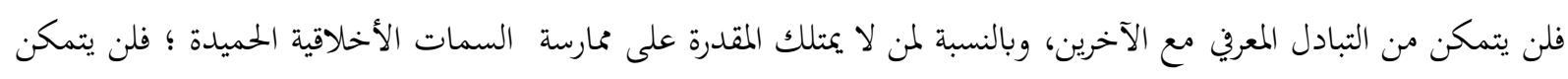

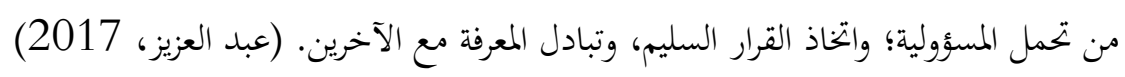

الخور الرابع: مناقشة المحاور

الإجابة عن السؤال الأول: ما دور أدبيات الرقمنة في المقاربة بين الرقمنة والعملية التعليمية؟

يُلاحظ أن خطة عمل المفوضية الأوروبية ركزت على جانبين للتعلم الرقمي، هما التقنيات الرقمية، والكفاءات الرقمية، وهذا

يتطلب سياسات تتناول محاور التعليم الرقمي كافة، وتتطلب إجراءات عملية، بحيث تشمل البنية التأسيسية (التحتية)، والاستراتيجيات التعليمية، وإدارة القيادات، ومهارات المعلم، ومهارات المتعلم والمحتوى التعليمي، والتقييم، والأطر القانونية الوطنية. كذلك ركزت خطة عمل المفوضية على أهمية التفكير الاستراتيجي في دمج التقنيات الرقمية في التعليم والتدريب، الأمر

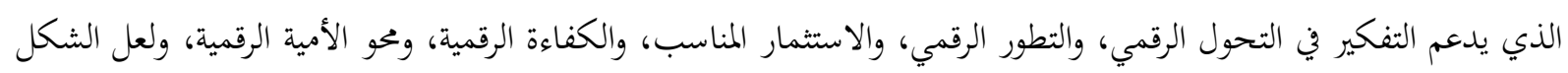
الآي يُوضح ذلك: 


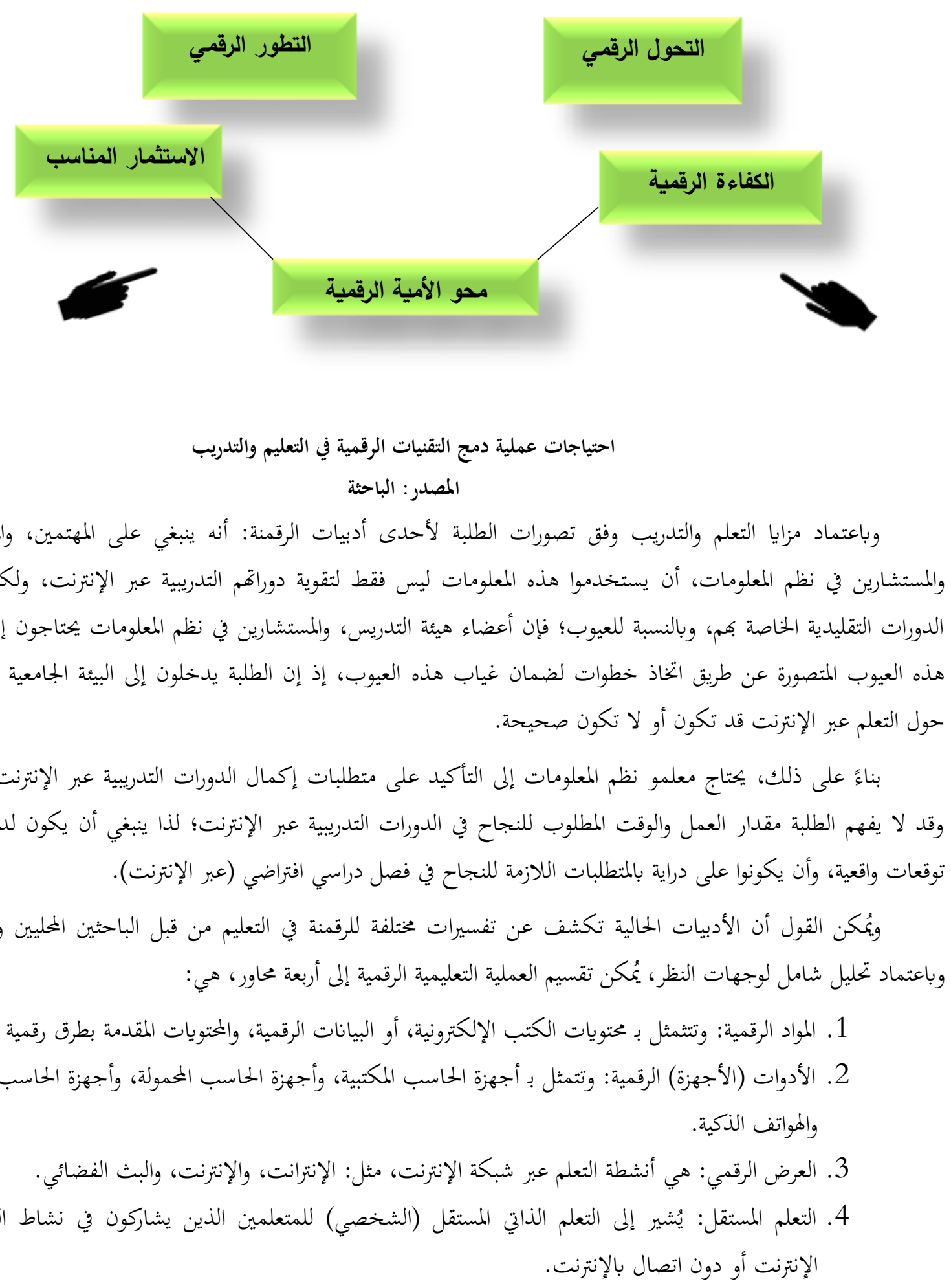



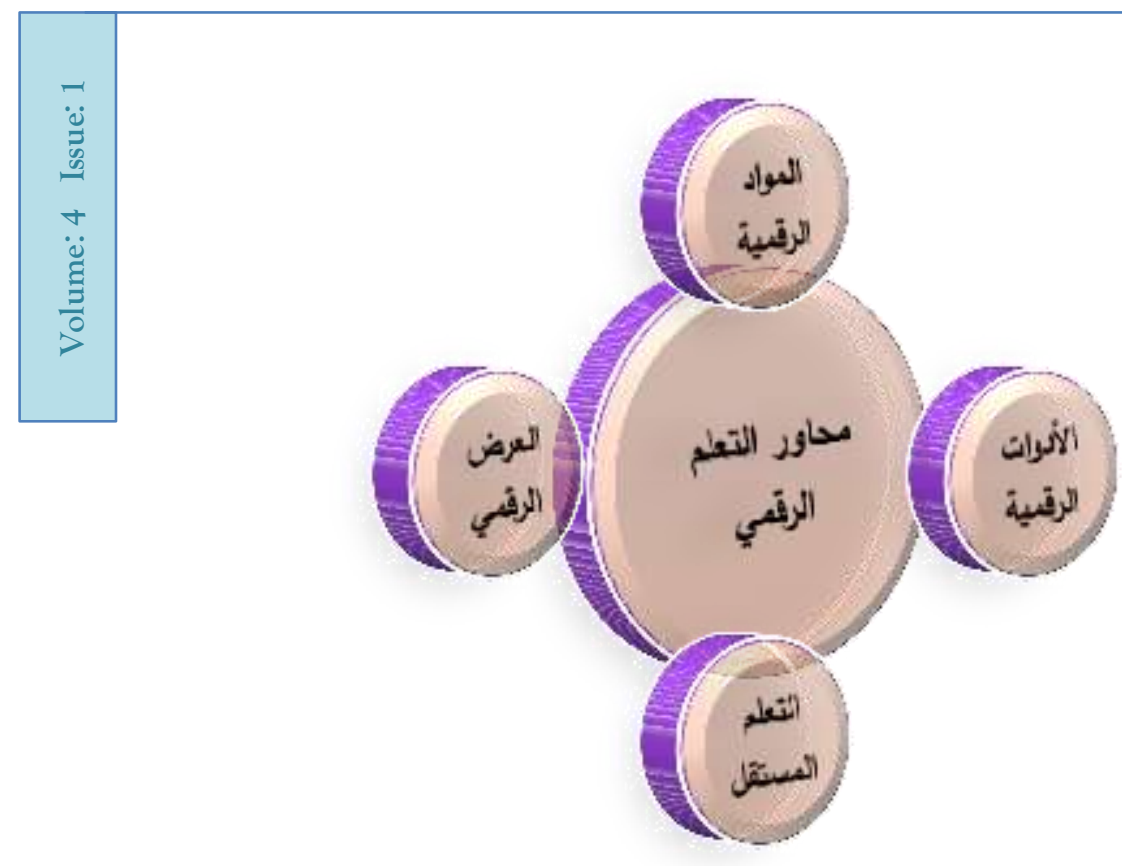

\section{محاور العملية التعليمية الرقمية}

المصدر: الباحثة

وفي ذات السياق، يُمكن استنتاج أن تعزيز فاعلية التعليم تتحقق، عندما تكون وظائف المنظومة التعليمية غنية، ومتنوعة لتكون قريبة من إدراك المتعلم، وبتذب المتعلم الذي يسجل عبر النظام الرقمي للتعليم، وفيما يتعلق بالمشكلات التي تواجه التعليم

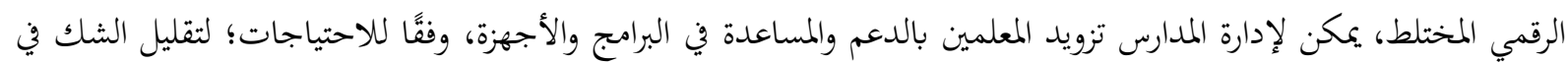
التعليم الرقمي، ويُمكنهم دمج المعلمين ذوي الاهتمامات لجمع الموارد ذات الصلة من الإنترنت وتطوير البرامج ذاتيًا، أو إنشاء صفحات الويب؛ لتعزيز عملية التعليم الرقمي .

فالمعلم هو مفتاح تطوير فاعلية التعليم الرقمي، فعندما يتبادل المعلمون خبرات التعليم مع أقراغم، أو مع الخبراء، أو المشاركة

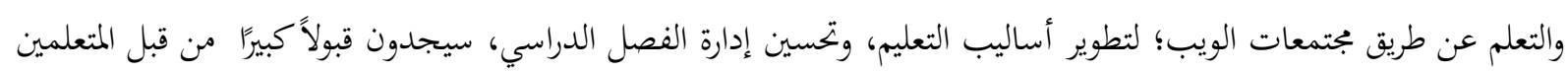

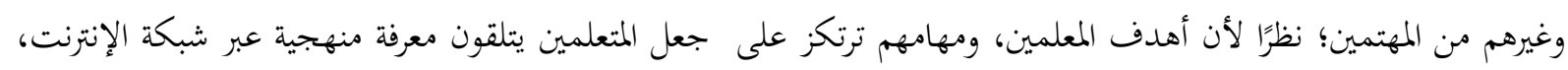

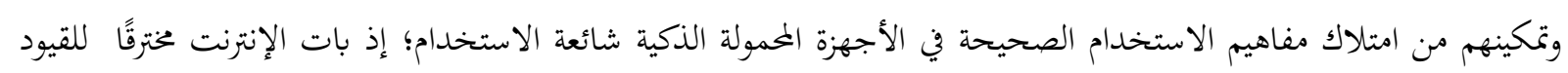

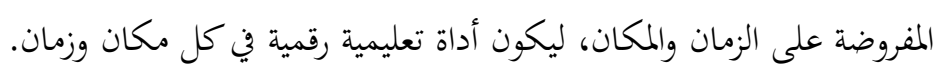
بناءً على ذلك، فإن تكلفة حلول التعليم الرقمي، واستدامتها، من الأسئلة الحاسمة التي لها نتائج قد تختلف كثيرًا لدى الباحثين حولما؛ إذ تعد الاستدامة وقابلية التوسع من الاهتمامات الكبيرة لواضعي السياسات والمهتمين في مجال التعليم، لا سيما

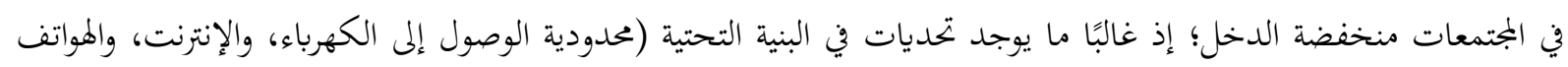
المحمولة) لأجزاء كبيرة من البلدان.

وبنظرة نقدية إلى إحدى الأدبيات التي ركزت على التعلم الرقمي باستخدام المنصات، يُمكن القول أن هذه المنصات

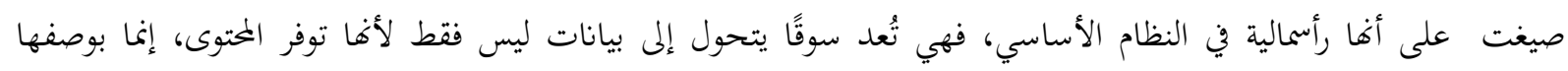

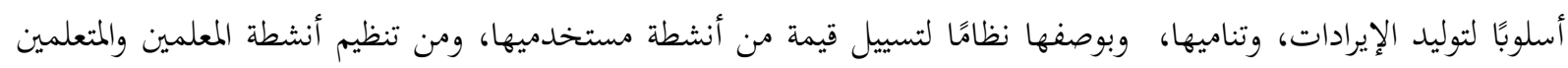

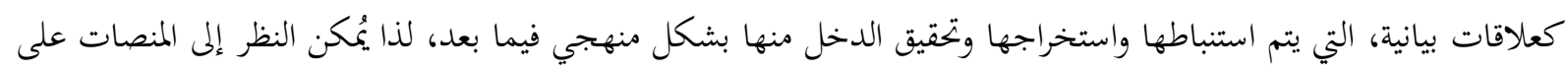

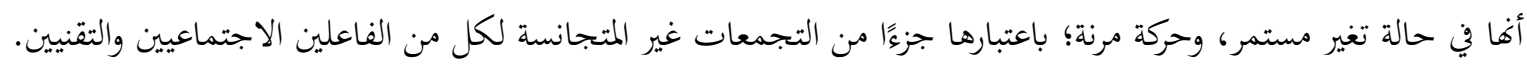


وفي مدى تأثير المنصات الرقمية في عملية التعليم وإعدادها؛ فهي تتدخل بقوة في عمليات الإعداد التعليمي، مع وجود

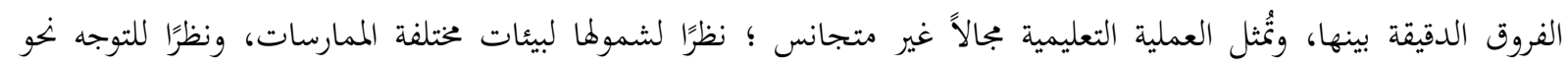

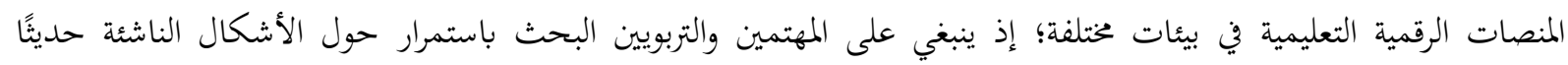
لمنصات التعليم الرقمي، وينبغي الاهتمام الحثيث في طبيعة المنصات الرقمية بوصفها أجهزة تعليمية رئيسة في إدارة العملية التعليمية،

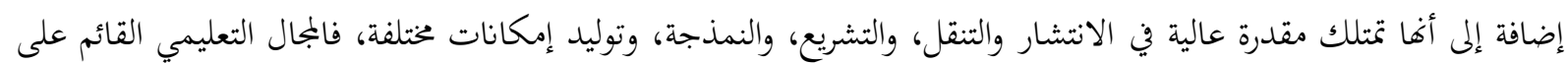

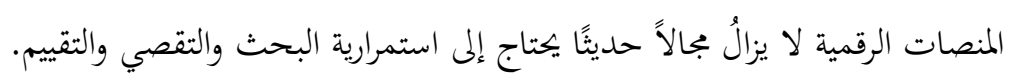

الإجابة عن السؤال الثاني: ما دور النظريات المفسرة للرقمنة في المقاربة بين الرقمنة والعملية التعليمية؟ يُككن القول أن نظرية جورج سيمنز عُرِفَت بمسميات كثيرة منها: الاتصالية، والشبكية، ونظرية التواصل الشبكي، ولعل

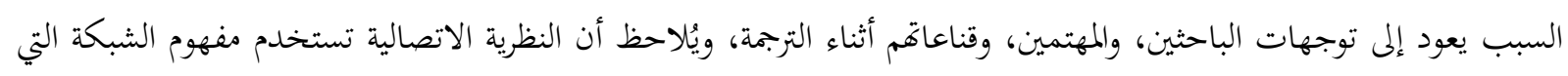

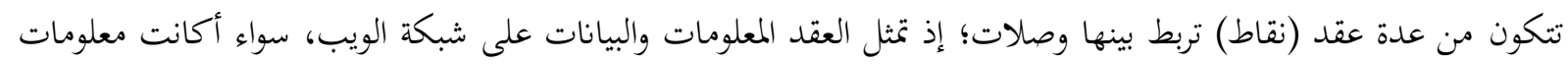

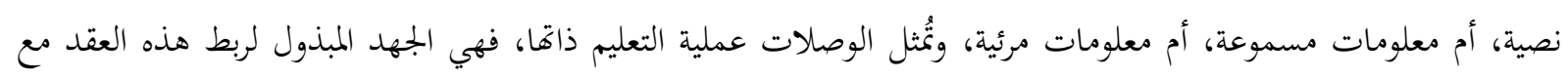
بعضها لتشكيل شبكة من المعارف الشخصية.

ويُمكن إبراز أهمية النظرية الاتصالية في التعليم الرقمي؛ إذ يحتل التعليم الرقمي أهمية كبيرة لدى النظرية الاتصالية عبر تطويع

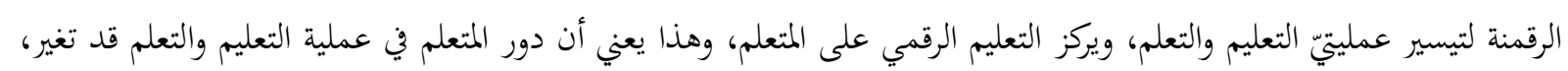

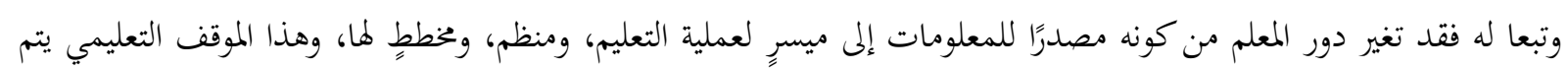

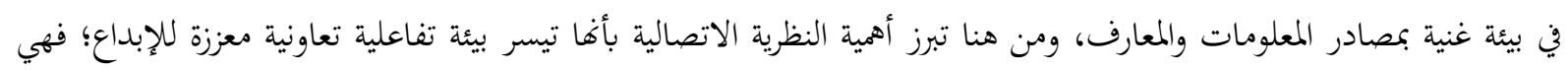

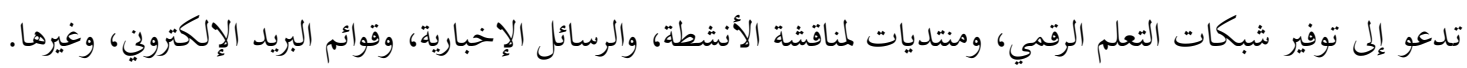
ويتضح أن التعليم في ضوء النظرية الاتصالية يتسم بالاستمرارية، والتعاونية، والتواصل، فهو ليس بثًا لمحتوى يتضمن مجموعة

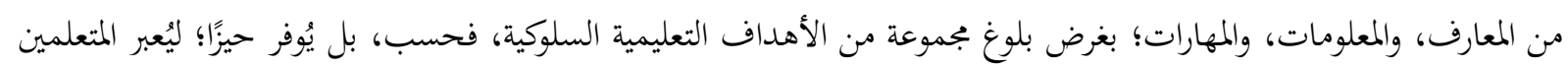

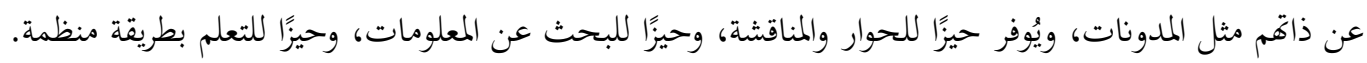
كما يتضح أن العملية التعليمية وفق نظرية العقول الخمسة تُمثل المقدرة على المشاركة لبناء المعرفة؛ لتكون العملية التعليمية

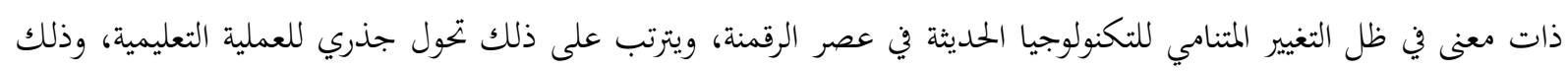

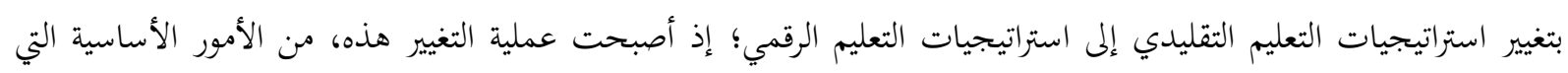
ينبغي اتخاذها في العصر الحالي ألا وهو العصر الرقمي.

بناءً على ما تقدم، فإن التعليم وفق نظرية العقول الخمسة يتطلب إعادة بناء المفاهيم العلمية بطريقة علمية في ذهن المتعلم،

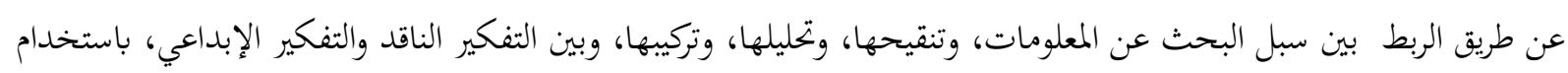

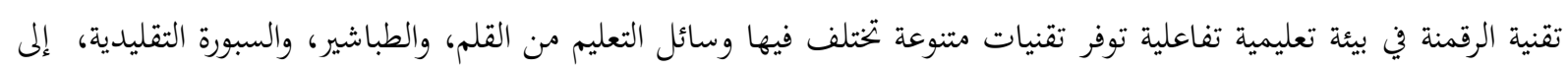
اللوح الذكي، والأجهزة المحمولة، والنقل الإلكتروين للوظائف التعليمية، والكتاب الإلكتروني (الرقمي). وفي ذات السياق، فقد تََّّل التغيير في استراتيجيات التعليم التقليدية، إلى استراتيجيات التعليم الرقمي مثل: استراتيجية

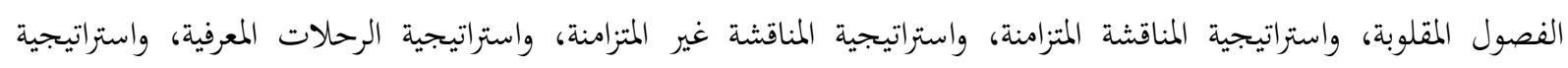

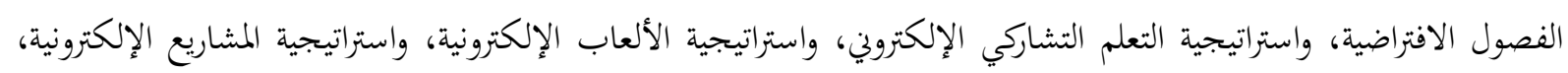

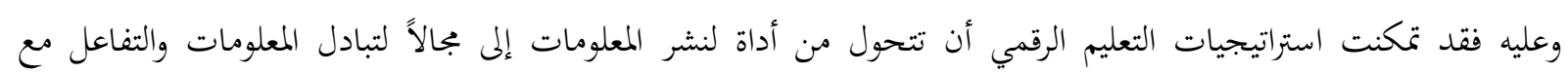


الآخرين، فلم يعد المتعلمين مستهلكين للإنترنت؛ عن طريق اكتساب المعلومات، بل أصبحوا مشاركين فاعلين فيها؛ نظرًا لدوررهم

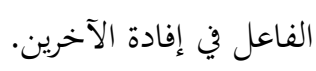

بناءً على ذلك، يُكن القول أن استخدام برنامج البلاك بورد) (Blackboard) يُسهم في تفاعل المعلم والمتعلم في

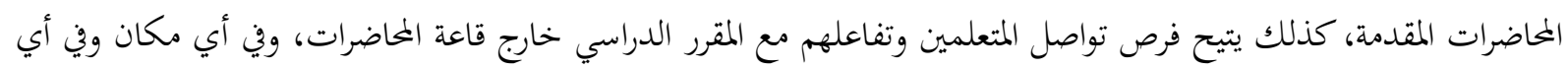

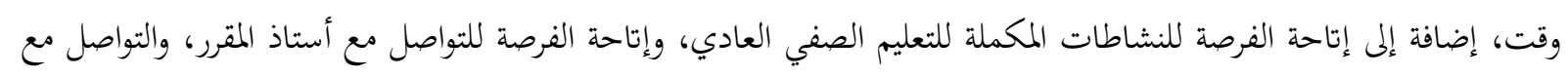

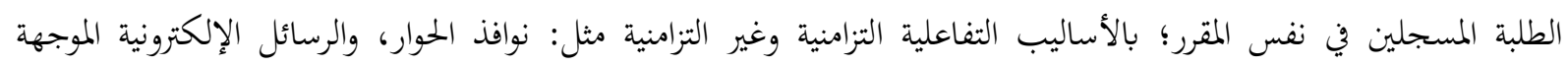

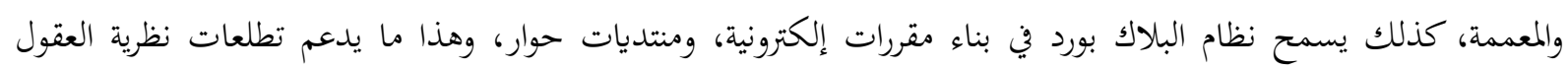

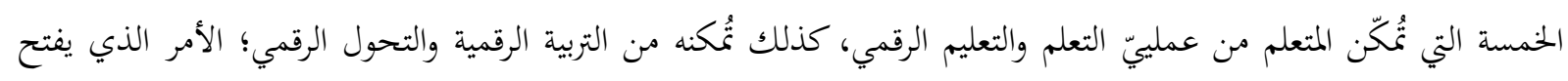

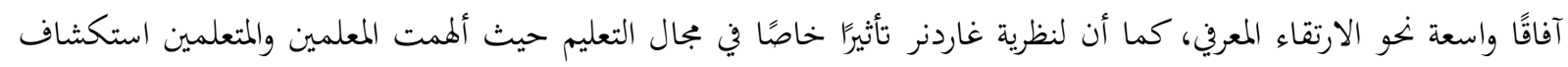

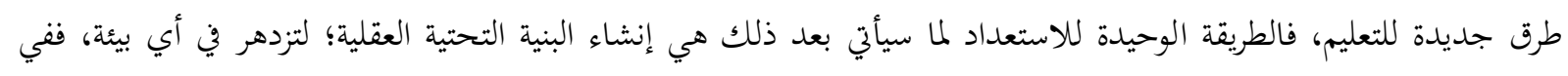

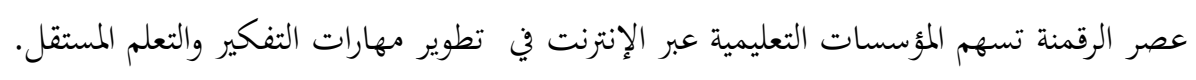

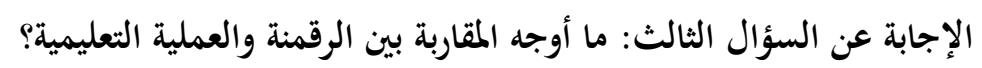

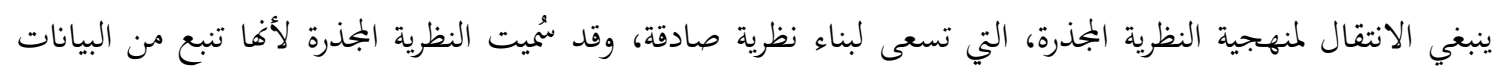

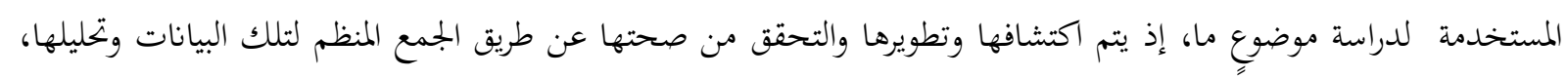

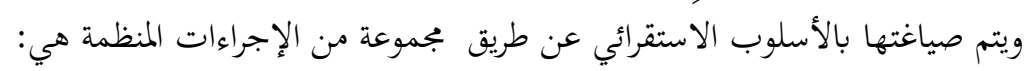

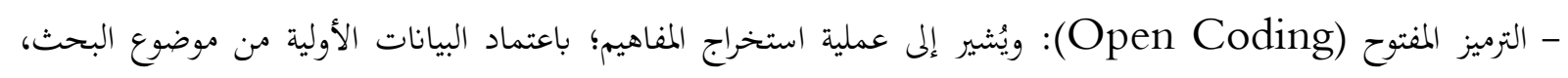

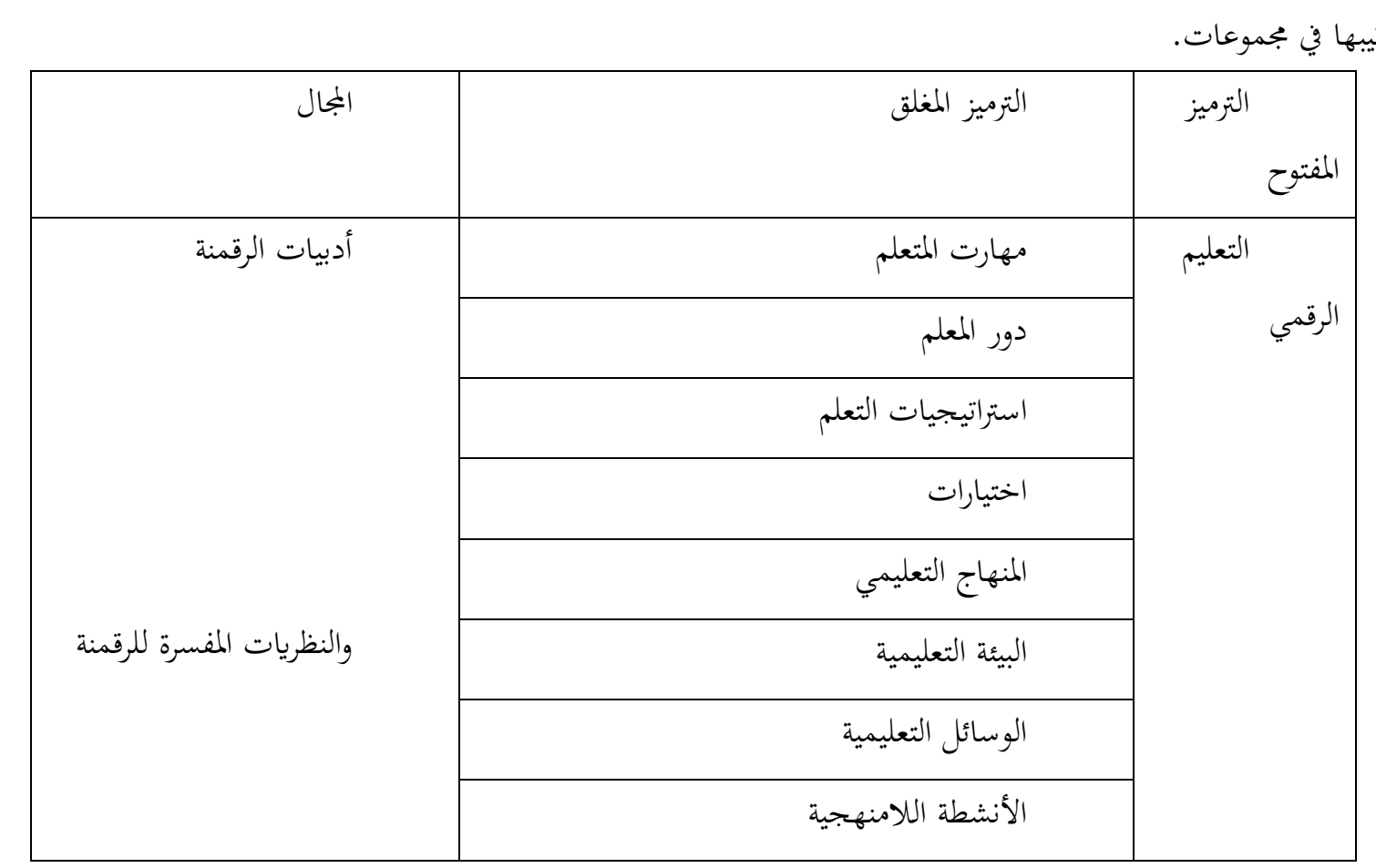

- الترميز المحوري (Axial Coding): يُشير إلى ترتيب البيانات بعضها مع بعض مرة أخرى؛ ليتم وضعها في مجموعات

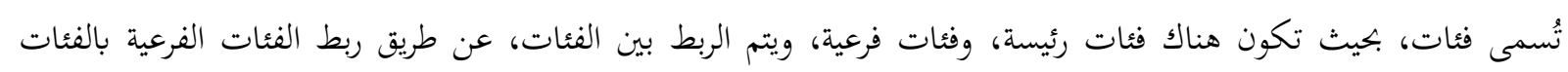




\begin{tabular}{|c|c|c|}
\hline الفئات الفر عية & الفئة الرئيسة & الترميز المحوري \\
\hline 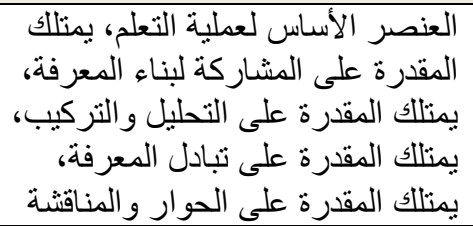 & المتعلم & تعليمية الرقمنة .... مقاربة \\
\hline 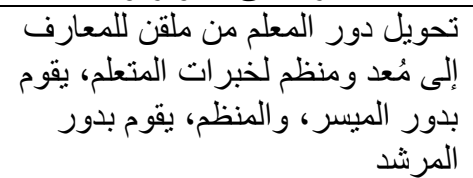 & المعلم & \\
\hline 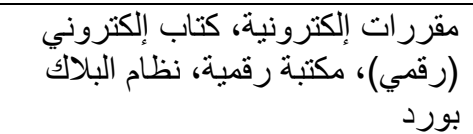 & المنهاج & \\
\hline 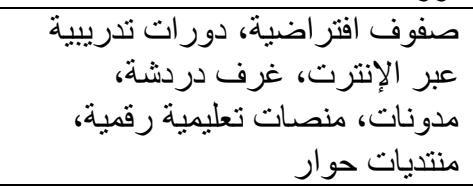 & بيئة تعليمية، صفي & \\
\hline ألوبي، ذكية، فيديو تعليمي، صفحة & وسائل تعليمية & \\
\hline 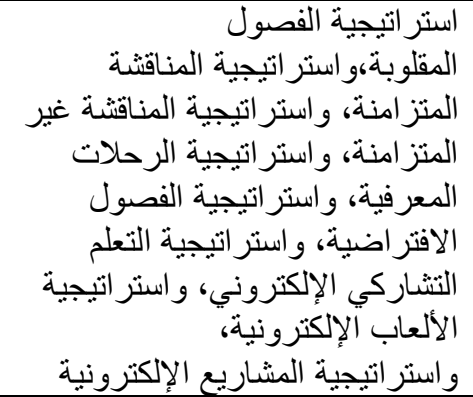 & استر اتيجيات التدريس & \\
\hline
\end{tabular}

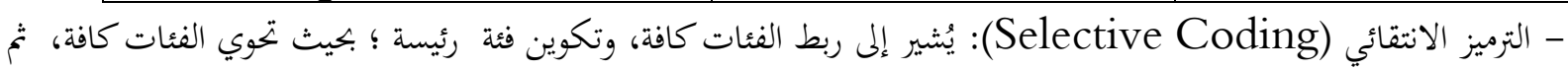

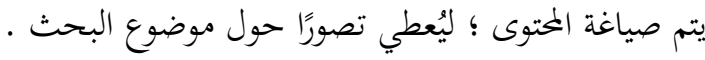

\begin{tabular}{|c|c|c|}
\hline ال المكونات & الفئة الرئيسة & الت الترميز الانتقائي \\
\hline المعلم و المتعلم & الرقمنة و العملية التعليمية & 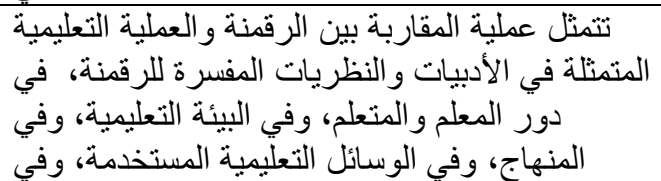 \\
\hline البيئة التعليمية & & 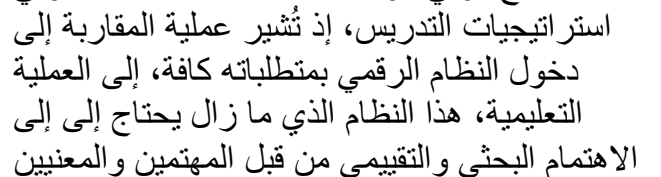 \\
\hline 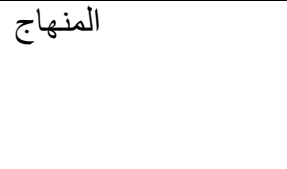 & & 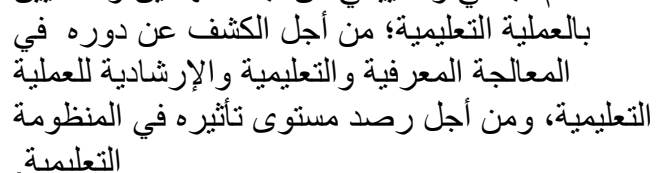 \\
\hline و استراتيجيات التندريسية، & & \\
\hline
\end{tabular}




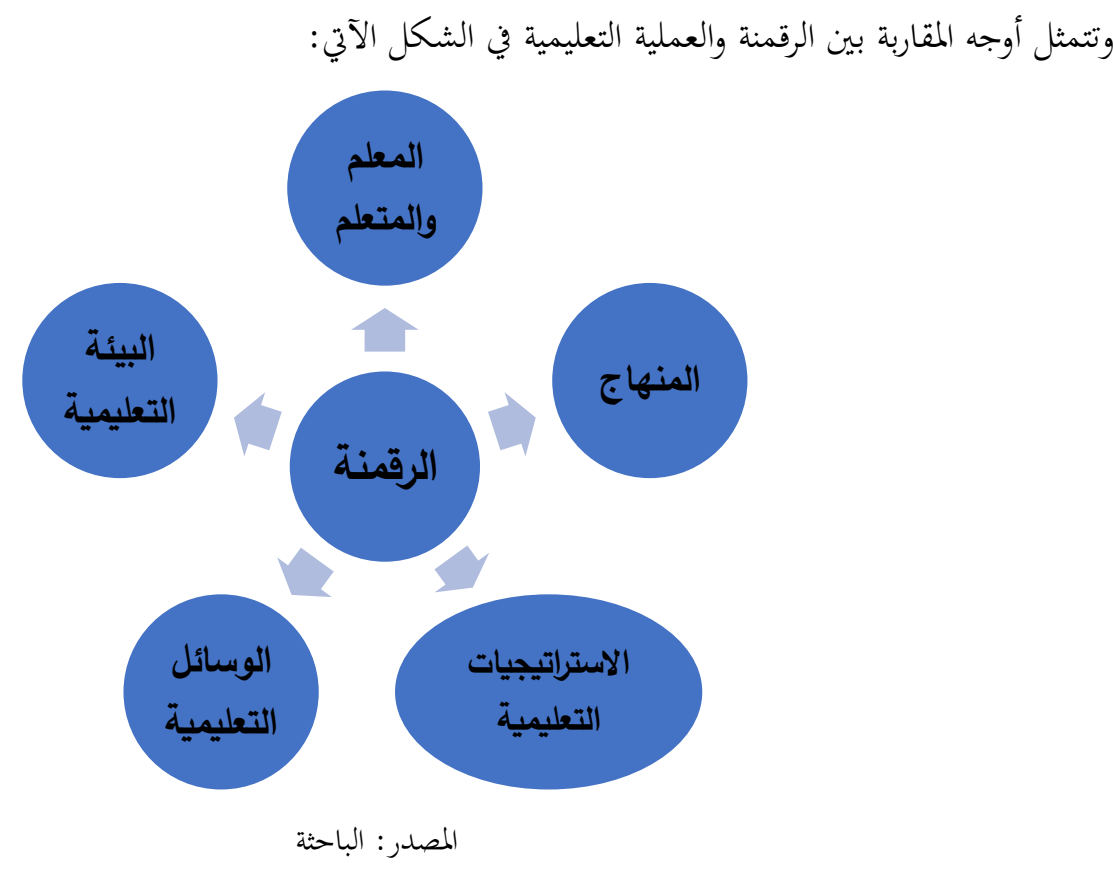

مؤشرات النتائج: تمثلت أوجه المقاربة بين الرقمنة والعملية التعليمية في المحاور الآتية: محور المعلم والمتعلم: أظهرت مؤشرات النتائج مقدرة الأجهزة الرقمية على تغيير دور المعلم والمتعلم في العملية التعليمية، فقد

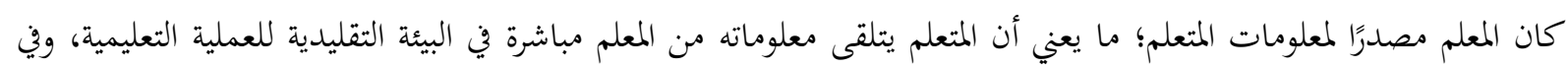

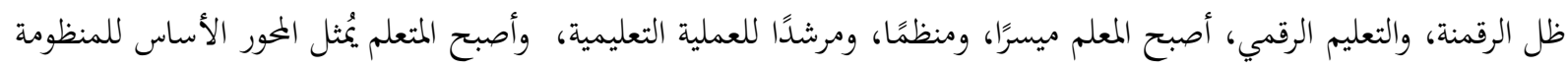

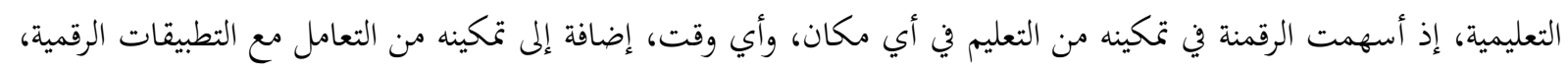
والتعلم عبرها. محور المنهاج: أظهرت مؤشرات النتائج مقدرة الرقمنة والتعلم الرقمي على استبدال الكتاب (المنهاج)، والمساقات التدريسية،

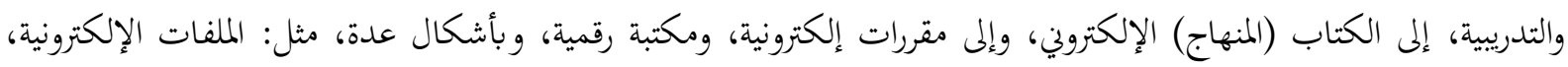

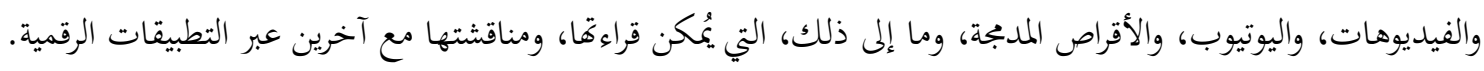
محور الوسائل التعليمية: أظهرت مؤشرات النتائج أن المقاربة بين الرقمنة والعملية التعليمية تكمن في استبدال الوسائل التعليمية التقليدية، مثل: السبورة، والقلم، والطباشير، وما شابه ذلك من الوسائل التعليمية التقليدية، إلى وسائل تعليمية رقمية ؛ مثل: نظام البلاك بورد، والألواح الذكية، والحاسب الآلي، وبرامجة المتنوعة، وتطبيقات الشبكة الإلكترونية. محور البيئة التعليمية: أظهرت مؤشرات النتائج أن المقاربة بين الرقمنة والعملية التعليمية تكمن في استبدال البيئة التعليمية

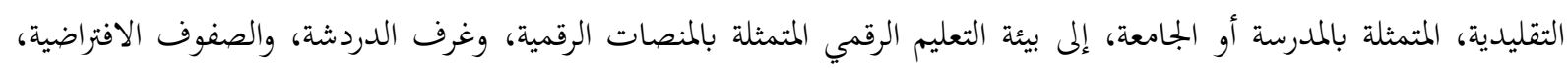
وتطبيقات الشبكة الإلكترونية المتنوعة.

محور الاستراتيجيات التعليمية: أظهرت مؤشرات النتائج أن المقاربة بين الرقمنة والعملية التعليمية تكمن في استبدال الاستراتيجيات التعليمية التقليدية، إلى استراتيجية الفصول المقلوبة، واستراتيجية المناقشة المتزامنة، واستراتيجية المناقشة غير المتزامنة، واستراتيجية الرحلات المعرفية عبر تطبيقات الشبكة، واستراتيجية الفصول الافتراضية، واستراتيجية التعلم التشاركي الإلكتروني،

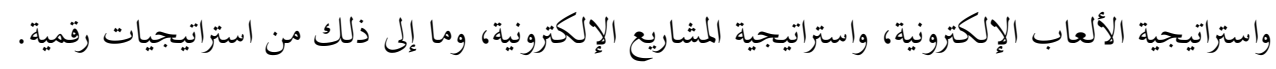




\section{التوصيات: يُمكن أن يُوصى بالآتي:}

1. أن يتفهم المسؤولون أهمية التخطيط لاستخدامات تقنية الرقمنة، مع إشراك الجهات الفاعلة في مجال التعليم، نظرًا لدخول الرقمنة في مجالات الحياة كافة، منها المجال التعليمي. 2. أن يتفهم المسؤولون أهمية تطوير البرامج التعليمية؛ لتحسين استجابتها للتغييرات التي تُحدثها الرقمنة في العملية التعليمية؛

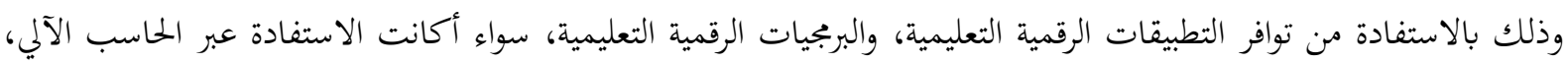
أم عبر الشبكة الإلكترونية. 3. أن يتفهم المعلمون أهمية التحاقهم بالبرامج التدريبية الخاصة بالرقمنة والعملية التعليمية؛ إذ يُسهم ذلك في الحدة من الممارسات السلبية جراء استخدام الأجهزة الرقمية ؛ الأمر الذي يدعم الدور الحقيقي للرقمنة في نجاح المسيرة التعليمية. 4. أن يتفهم المسؤولون أهمية التعاون، والمشاركة، للقطاعات كافة، في تجهيز البهاء البنية التأسيسية (التحتية) للتعليم الرقمي؛ نظرًا لانتشاره عبر التطبيقات الرقمية كافة، واعتماده لدى الدول المتقدمة . 5. أن يهتم الخبراء والباحثون في إجراء مزيدٍ من البحوث حول الرقمنة والعملية التعليمية، ويُمكن اقتراح بعض المسميات للبحوث المقترحة كالآتي: - - دور الرقمنة في تعزير العملية التعليمية من وجهة نظر المعلمين. - الرقمنة وأثرها في العملية التعليمية من وجهة نظر الخبراء. - الآثار الإيجابية والسلبية الناجمة عن استخدام تقنية الرقمنة في العملية التعليمية من وجهة نظر الطلبة.

يُمكن صياغة النظرية المجذرة لنهج هذا البحث، بالتأكيد على وجود علاقة ترابطية وثيقة بين الرقمنة والعملية التعليمية، إذ

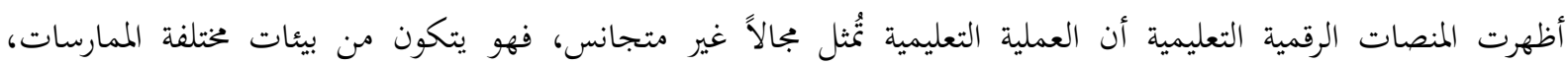

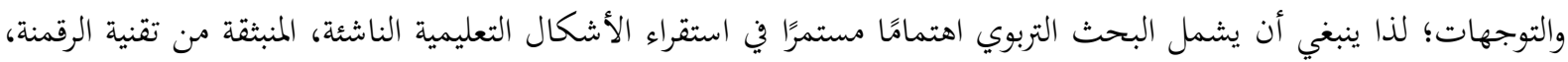
وما يتصل بذلك حول الكشف عن الإمكانيات التعليمية، ورصدها. وفي ذات السياق، يُمكن القول أن التقنية الرقمية، وما تحمله من سمات مستجدات العصر ومتطلباته، قد بتحد مسيرتا عائقًا لدى بعض المجتمعات التي ترفض التغيير، وتُثقله بالسلبيات، ومن منظور الأدبيات والنظريات المفسرة للرقمنة في التعليم؛ فإنه ومنه

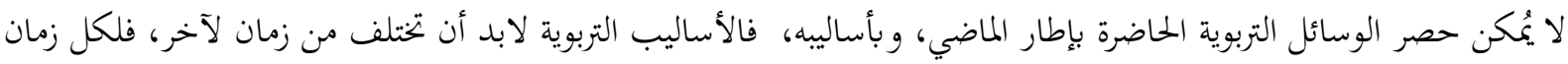

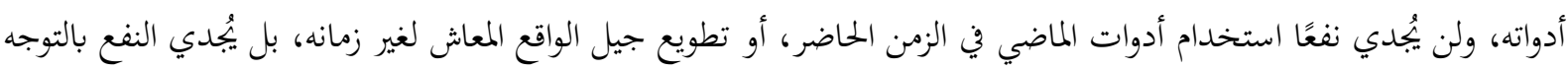
إلى الاستثمار في العقول البشرية، عن طريق استخدام ما يناسب زمافم من أساليب وأدوات؛ لتحقيق أهداف العملية التعليمية. 


$$
\begin{aligned}
& \text { مصطفى، إبراهيم ـ الزيات، أحمد حسن. عبد القثادر، حامد . والنجار، محمد علي. (بدون تاريخ). المعجم الوسيط. اسطنبول: } \\
& \text { المكتبة الإسلامية للطباعة والنشر والتوزيع. } \\
& \text { الفقيه،أحمد حسن (2017). تصميم البحث النوعي في البمال التربوي مع التركيز على بحوث تعليم اللغة العربية. المجلة الدولية }
\end{aligned}
$$

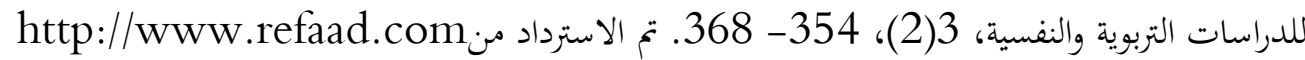

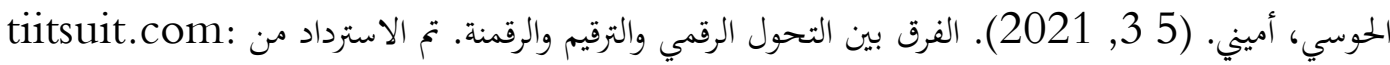

$$
\begin{aligned}
& \text { https://www.tiiitsuit.com/ } \\
& \text { الودية، رانية طه. (8 8, 2018). التربية الرقمية. تم الاسترداد من }
\end{aligned}
$$

http://www.lahaonline.com/articles/view/56031.htm.

$$
\text { صبري، رشا السيد. (2020). برنامج مقترح قائم علي نظريتي تعلم لعصر الثورة الصناعية الرابعة. المجلة التربوية/ جامعة عين } 440 \text { - 540. }
$$

https://webinar.attaa.sa/files/webinars: المفضي، سارة محمد. (2021). التحول الرقمي. تم الاسترداد من https://webinar.attaa.sa/files/webinars

$$
\text { الزعبوط، سمية عيد. (2021). تقنية الذكاء الاصطناعي: مقاربة تعليمية. المؤتمر العلمي الدولي الثاني عشر، بعنوان (بحوث }
$$

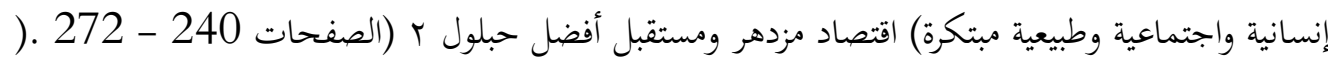

$$
\text { شn Line: }
$$

https://azimyouth.org/: يوث، عزام. ( 2021). مفهوم العملية التعليمية. تم الاسترداد من مورات العبية

https://azimyouth.org/

حمودة،علي · وحسين، محمد. (2019). فاعلية بنية محتوى البوابات إللكترونية في. بجلة البحوث الإلعالمية، 33 - 76. الزبيدي، كمال. (6 12, 2017) 2017). المقاربة . عبد العزيز، منى طه. (2017). الجودة في التعليم الإلكتروني: الخبرات العالمية. بجلة المعرفة التربوية، 80 - 100.

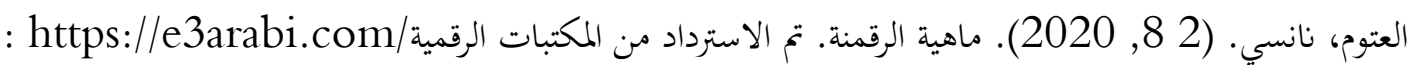
الحلفاوي، وليد سالم. (2011). التعلم الإلكتروني: تطبيقات مستحدثة. القاهرة: دار الفكر العربي للنشر والتوزيع.

\section{References}

Alexander, M. W., Truell, A. D., \& Zhao, J. J. (2012). “ EXPECTED ADVANTAGES AND DISADVANTAGES OF ONLINE LEARNING: PERCEPTIONS FROM COLLEGE STUDENTS WHO HAVE NOT TAKEN ONLINE COURSES”. Issues in Information Systems, 13(2), 193- 200.

Commission, E. (2021). Digital Education. Retrieved from Action Plan about Resetting Education and Training for Digital Age: https://ec.europa.eu/education/education-in-theeu/digital-education-action-plan_en

Decuypere, M., Landri, P., \& Grimaldi, E. (2021). Critical studies of digital education platforms. Critical Studies in Education Journal, 62(1), 1 -16.

Erickson, F. (1986). Qualitative methods in research on teaching. In M. Wittrock (Ed.), Handbook of research on teaching (3.ed ed.). New York: New York Macmillan. 
Kimberly, S. (2014). Five Minds for the Future: Shaping the Future through Education. Athletic Training Education Journal, , 9(1.(

Lim, A., Wong, S., Boon, L., \& Tan, A. (2009). Developing The Five Minds of the future, Villach: Conference I.C.L, Austria. Conference I.C.L, Austria. Austria: http://www.iclconference.org/di/proceedings/2009/program/pdf/Contribution_133.pdf.

Ming, L. H., Huang, C. C., \& Kuang, L. S. (2017). A Study of the Effects of Digital Learning on Learning Motivation and Learning Outcome. EURASIA Journal of Mathematics Science and Technology Education., 13(7), 3554 - 3564.

Siemens, G. (2020). wikipedia. Retrieved from https://en.wikipedia.org/wiki/George_Siemens. UNICEF. (2021). Effectiveness of Digital Learning Solutions Improve Educational Outcomes: A Review of the Evidence. Retrieved from https:/www.unicef.org/media/103246/.pdf: https://www.unicef.org/media/103246/.pdf 